\title{
Third-order perturbation expansion of the two-point correlation function of the dissipative quantum $\varphi^{4}$ theory
}

\author{
Panagiotis V. Alatas $\odot,{ }^{1}$ Vlasis G. Mavrantzas $\odot,{ }^{1,2, *}$ and Hans Christian Öttinger ${ }^{3}$ \\ ${ }^{1}$ Department of Chemical Engineering, University of Patras \& FORTH-ICE/HT, GR 26504 Patras, Greece \\ ${ }^{2}$ Particle Technology Laboratory, Department of Mechanical and Process Engineering, ETH Zürich, \\ CH-8092 Zürich, Switzerland \\ ${ }^{3}$ Polymer Physics, Department of Materials, ETH Zürich, CH-8093 Zürich, Switzerland
}

(Received 3 July 2021; accepted 23 August 2021; published 8 October 2021)

\begin{abstract}
We analytically calculate the perturbation expansion of the two-point correlation function (propagator) of the scalar, dissipative quantum $\varphi^{4}$ theory up to third order in the interaction parameter $\lambda$. The calculations are carried out with two different methods. The first is based on a brute force computation of all interactions appearing in the perturbation expansion term by term at the given order of the perturbation, supported by the use of a symbolic mathematical code. The second uses an efficient perturbation scheme which allows one to hierarchically organize terms within a given order in the perturbation expansion based on the number of occurrences of the free evolution operator $\mathcal{R}_{s}^{\text {free}}$; in addition, it allows one to utilize information from already computed lower-order terms in the calculation of higher-order terms. The two methods yield the same result. In the limit of zero dissipation (zero friction coefficient $\gamma$ ), our result coincides with that obtained from the Lagrangian approach in $D=d+1$ dimensions ( $d$ denotes the number of space dimensions, while $D$ includes the time dimension) with the use of $D$-dimensional vectors, re-expressed in terms of only $d$-dimensional space integrals by integrating out the zero component. We have also verified that the value of the critical coupling constant $\lambda^{*}$ as a function of space dimensionality $d$, which can be used to evaluate perturbation expansions at the physical length scale $\ell_{m}$, comes out correctly from our thirdorder calculation.
\end{abstract}

DOI: $10.1103 /$ PhysRevD.104.076007

\section{INTRODUCTION}

In quantum field theories, Feynman diagrams involving closed loops require integration over all possible combinations of energy and momentum of the virtual particles traveling around the loop and are often divergent. To treat such divergences, renormalization of the parameters of the theory is required [1-3]. Behind renormalization is the problem of the proper, successive elimination of degrees of freedom in the effort to bridge a wide range of length scales and, in relativistic quantum field theory, clearly also of time scales [4-6]. Renormalization specifies relationships between parameters in the theory whose values at large length scales are different from the values at short length scales.

Considering irreversibility to be an intrinsic feature of any quantum field theory, since the divergences arise from spontaneous particle creation and annihilation (processes

\footnotetext{
vlasis@chemeng.upatras.gr, vlasios.mavrantzas@mat.ethz.ch
}

Published by the American Physical Society under the terms of the Creative Commons Attribution 4.0 International license. Further distribution of this work must maintain attribution to the author(s) and the published article's title, journal citation, and DOI. that are far beyond our mechanistic control being too fast and too local) [6], it is natural to introduce a dissipative smoothing mechanism which can provide ultraviolet regularization, even in the thermodynamic limit. The corresponding friction parameter is related to a small characteristic length scale such that phenomena below this scale cannot be resolved by the theory, and only phenomena above this characteristic length scale can be addressed [4].

The slow, large-scale, low-energy features of a dissipative quantum system are most naturally described by a thermodynamic quantum master equation for the evolution of the density matrix in Fock space [7,8], which, by construction, is driven to equilibrium. The quantum master equation describes collisions of free particles (field quanta) as well as the interaction with a heat bath representing small-scale features. The dissipative coupling to the bath is assumed to be very weak (except at short length scales) implying that short-scale features are erased very rapidly whereas largescale ones remain unaffected. In general, thermodynamic quantum master equations are nonlinear in the density matrix [9-11], a feature which clearly distinguishes them from the popular Lindblad master equations [12].

Quantum master equations for dissipative systems involve decay rates $\gamma_{k}$ which are negligible for small $k$ 
but increase rapidly for large $k$. The following concrete functional form for $\gamma_{k}$ has been proposed [4]:

$$
\gamma_{k}=\gamma_{0}+\gamma k^{4},
$$

where the parameter $\gamma_{0}$ has been added so that also the state with $k=0$ is subject to some dissipation. As we are eventually interested in the limit $\gamma_{k} \rightarrow 0$, the parameters $\gamma_{0}$ and $\gamma$ should be regarded as small. Within the natural units convention ( $\hbar=c=1$, where $\hbar$ is the reduced Planck constant and $c$ the speed of light), $\gamma_{0}$ has units of mass and $\gamma$ units of mass ${ }^{-3}$. A small characteristic length scale associated with dissipation can hence be introduced as $\ell_{\gamma}=\gamma^{1 / 3}$.

The dissipative coupling to the heat bath in the thermodynamic quantum master equation provides a proper highenergy (ultraviolet) cutoff, thus a regularization of the theory for dynamic properties. As we will also see below, in the intermediate mathematical calculations involved in computing correlation functions, the regularizing effect of dissipation is typically contained in the use of modified frequencies $\breve{\omega}_{k}=\omega_{k}-\mathrm{i} \gamma_{k}$ and their complex conjugates. Clearly, if one neglects irreversible contributions to dynamics, the regularizing effect of dissipation is lost. Similarly, if no dynamic regularization is needed, one can perform the limit of vanishing friction before doing any calculations.

As explained in [4], thermodynamic dissipative regularization is attractive also for another reason: the use of a thermodynamically consistent quantum master equation guarantees a controlled long-time behavior due to the robust thermodynamic structure of the evolution equations that is not shared by the usual regularization methods. As an important consequence, correlation functions can be rigorously reformulated in terms of free vacuum expectation values. In the case of the scalar theory with quartic interactions ( $\varphi^{4}$ theory), in particular, two-point and four-point correlation functions are of interest. The first includes two fields and serves to define the propagator, while the second includes four fields and corresponds to the effective interaction vertex. The latter has helped [4] compute the fixed point value of the dimensionless coupling constant [3].

In this work, we apply the dissipative quantum field theory [4] to calculate the two-point correlation function (propagator) of the scalar $\varphi^{4}$ theory up to third order in the interaction parameter $\lambda$. In Sec. II, we introduce the basic equations and we highlight two different methodologies for evaluating the two-point correlation function in the context of the dissipative theory, both leading to the same exact result. The final result for the propagator up to third order is derived in Sec. III. In Sec. IV, we demonstrate the equivalence, in the limit of zero dissipation, with the Lagrangian approach to quantum field theory starting from $D=d+1$ space-time dimensions. In Sec. V, we propose a regularization leading to parameters that avoid divergent contributions and hence to a finite expression. In Sec. VI, we discuss the calculation of the critical value $\lambda^{*}$ of the coupling constant $\lambda$ of the theory. We conclude with Sec. VII summarizing the most important results of our work.

\section{DISSIPATIVE SCALAR FIELD THEORY}

The dissipative scalar field theory is based on one kind of bosonic quanta associated with the creation and annihilation operators $a_{k}^{\dagger}$ and $a_{k}$, respectively. For massive quanta, the free Hamiltonian reads

$$
H^{\mathrm{free}}=\sum_{k \in K^{d}} \omega_{k} a_{k}^{\dagger} a_{k}
$$

with the following relativistic energy-momentum relation for a particle with mass $m$ and momentum $k=|\boldsymbol{k}|$ :

$$
\omega_{k}=\sqrt{m^{2}+k^{2}} .
$$

The quartic interactions are described by the Hamiltonian

$$
\begin{aligned}
H^{\text {coll }}= & \frac{\lambda}{96} \frac{1}{V} \sum_{\boldsymbol{k}_{1}, \boldsymbol{k}_{2}, \boldsymbol{k}_{3}, \boldsymbol{k}_{4} \in K^{d}} \frac{\delta_{\boldsymbol{k}_{1}+\boldsymbol{k}_{2}+\boldsymbol{k}_{3}+\boldsymbol{k}_{4}, \mathbf{0}}}{\sqrt{\omega_{k_{1}} \omega_{k_{2}} \omega_{k_{3}} \omega_{k_{4}}}} \\
& \times\left(a_{-\boldsymbol{k}_{1}} a_{-k_{2}} a_{-\boldsymbol{k}_{3}} a_{-\boldsymbol{k}_{4}}+4 a_{\boldsymbol{k}_{1}}^{\dagger} a_{-\boldsymbol{k}_{2}} a_{-\boldsymbol{k}_{3}} a_{-k_{4}}\right. \\
& \left.+6 a_{\boldsymbol{k}_{1}}^{\dagger} a_{\boldsymbol{k}_{2}}^{\dagger} a_{-\boldsymbol{k}_{3}} a_{-\boldsymbol{k}_{4}}+4 a_{\boldsymbol{k}_{1}}^{\dagger} a_{\boldsymbol{k}_{2}}^{\dagger} a_{\boldsymbol{k}_{3}}^{\dagger} a_{-\boldsymbol{k}_{4}}+a_{\boldsymbol{k}_{1}}^{\dagger} a_{\boldsymbol{k}_{2}}^{\dagger} a_{\boldsymbol{k}_{3}}^{\dagger} a_{\boldsymbol{k}_{4}}^{\dagger}\right) \\
& +\frac{\lambda^{\prime}}{4} \sum_{k \in K^{d}} \frac{1}{\omega_{k}}\left(a_{\boldsymbol{k}} a_{-\boldsymbol{k}}+2 a_{\boldsymbol{k}}^{\dagger} a_{\boldsymbol{k}}+a_{\boldsymbol{k}}^{\dagger} a_{-k}^{\dagger}\right)+\lambda^{\prime \prime} V
\end{aligned}
$$

where we have introduced a $d$-component version of Kronecker's $\delta$ symbol and the parameters

$$
\lambda^{\prime}=\lambda \frac{1}{V} \sum_{k \in K^{d}} \frac{1}{4 \omega_{k}}, \quad \lambda^{\prime \prime}=\frac{1}{2} \lambda\left(\frac{1}{V} \sum_{k \in K^{d}} \frac{1}{4 \omega_{k}}\right)^{2} .
$$

The interaction parameter $\lambda$ characterizes the strength of the quartic interaction.

The zero-temperature $\left[\beta \rightarrow \infty, \beta=1 /\left(k_{B} T\right)\right]$ quantum master equation summarizing Hamiltonian and irreversible dynamics is given by

$$
\begin{aligned}
\frac{d \Delta \rho_{t}}{d t}= & -\mathrm{i}\left[H, \Delta \rho_{t}\right] \\
& +\sum_{k \in K^{d}} \gamma_{k}\left(2 a_{k} \Delta \rho_{t} a_{k}^{\dagger}-a_{k}^{\dagger} a_{k} \Delta \rho_{t}-\Delta \rho_{t} a_{k}^{\dagger} a_{k}\right) \\
& -\sum_{k \in K^{d}} \frac{\gamma_{k}}{\omega_{k}}\left(\left[a_{k}, \Delta \rho_{t}\left[a_{k}^{\dagger}, H^{\text {coll }}\right]\right]+\left[a_{k}^{\dagger},\left[a_{k}, H^{\text {coll }}\right] \Delta \rho_{t}\right]\right),
\end{aligned}
$$

where $\Delta \rho_{t}=\rho_{t}-\rho_{\text {eq }}$ denotes the deviation from the equilibrium (ground-state) density matrix, $H=H^{\text {free }}+H^{\text {coll }}$ is the full Hamiltonian, and $\gamma_{k}$ is the decay rate defined by 
Eq. (1). The first term on the right-hand side of Eq. (6) describes the reversible contribution to time evolution, while the other terms are of irreversible nature. The general thermodynamic master equation for a quantum system in contact with a heat bath with temperature $T$ is shown in [11]. Any ground state of the interacting theory can be written as $|\Omega\rangle=|0\rangle+|\omega\rangle$, where $|\omega\rangle$ has no component along the vacuum state $|0\rangle$ and can be evaluated as

$$
|\omega\rangle=\sum_{n=1}^{\infty}\left[-R_{0}^{\mathrm{free}} H^{\mathrm{coll}}\right]^{n}|0\rangle .
$$

In Eq. (7), $R_{0}^{\text {free }}$ is a self-adjoint operator defined as $R_{0}^{\text {free }}=\left(H^{\text {free }}\right)^{-1} P_{0}=P_{0}\left(H^{\text {free }}\right)^{-1}=P_{0}\left(H^{\text {free }}\right)^{-1} P_{0}$, where $H^{\text {free }}$ is the free Hamiltonian defined by Eq. (2) and $P_{0}$ the projector operator $P_{0}=1-|0\rangle\langle 0|$ suppressing the ground state. Throughout this work, we use natural units with $\hbar=c=1$, where $\hbar$ is the reduced Planck constant and $c$ the speed of light.

On the basis of the zero-temperature quantum master equation, Eq. (6), the following Laplace-transformed twopoint correlation function of the operators $A_{1}$ and $A_{2}$ is considered:

$$
\tilde{C}_{s}^{A_{2} A_{1}}=\operatorname{tr}\left[A_{2} \mathcal{R}_{s}\left(A_{1} \rho_{\mathrm{eq}}\right)\right]
$$

where $\operatorname{tr}\left(A_{1} \rho_{\text {eq }}\right)=0$. In this work, $A_{1}$ and $A_{2}$ are chosen to be the Fourier components of the free particle field

$$
A_{1}=\varphi_{k}=\frac{1}{\sqrt{2 \omega_{k}}}\left(a_{k}^{\dagger}+a_{-k}\right)
$$

and

$$
A_{2}=\varphi_{-k}=\frac{1}{\sqrt{2 \omega_{k}}}\left(a_{-k}^{\dagger}+a_{k}\right) .
$$

In Eq. (8), $\mathcal{R}_{s}$ is the Laplace-transformed evolution superoperator $\{$ see Eq. (1.82) of [4]\} for which we have the following perturbation expansion around $\mathcal{R}_{s}^{\text {free }}$ :

$$
\begin{aligned}
\mathcal{R}_{s}= & \mathcal{R}_{s}^{\text {free }}+\mathcal{R}_{s}^{\text {free }} \mathcal{L}^{\text {coll }} \mathcal{R}_{s}^{\text {free }} \\
& +\mathcal{R}_{s}^{\text {free }} \mathcal{L}^{\text {coll }} \mathcal{R}_{s}^{\text {free }} \mathcal{L}^{\text {coll }} \mathcal{R}_{s}^{\text {free }}+\cdots
\end{aligned}
$$

Within the simplified irreversible dynamics (SID) assumption [4] (namely, that we neglect terms that will eventually disappear in the limit $\gamma \rightarrow 0$ at an early stage of the calculation), the linear collision superoperator $\mathcal{L}^{\text {coll }}$ is given by

$$
\mathcal{L}^{\text {coll }}(\rho)=-\mathrm{i}\left[H^{\text {coll }}, \rho\right]
$$

Regarding the action of the free evolution operator $\mathcal{R}_{s}^{\text {free }}$ on an operator $X$ of the general form

$$
X=a_{\boldsymbol{k}_{1}}^{\dagger} \ldots a_{\boldsymbol{k}_{n}}^{\dagger}|0\rangle\langle 0| a_{\boldsymbol{k}_{n}^{\prime}} \ldots a_{\boldsymbol{k}_{1}^{\prime}},
$$

in the context of the SID assumption, we have

$\mathcal{R}_{s}^{\mathrm{free}}(X)=\frac{X}{s+\mathrm{i}\left(\breve{\omega}_{k_{1}}+\ldots+\breve{\omega}_{k_{n}}-\breve{\omega}_{k_{1}^{\prime}}^{*}-\ldots-\breve{\omega}_{k_{n}^{\prime}}^{*}\right)}$.

In the special case where the free evolution operator $\mathcal{R}_{s}^{\text {free }}$ acts directly on the dual state $|0\rangle\langle 0|$, the result is $\mathcal{R}_{s}^{\text {free }}(|0\rangle\langle 0|)=1 / s$. In the denominator of Eq. (13), the modified frequencies $\breve{\omega}_{k_{j}}$, along with their complex conjugates $\breve{\omega}_{k_{j}}^{*}$, are given by

$$
\breve{\omega}_{k_{j}}=\omega_{k_{j}}-\mathrm{i} \gamma_{k_{j}}
$$

and contain the regularizing effect of dissipation. Similar to $\mathcal{R}_{s}^{\text {free }}$ is the action of the self-adjoint operator $R_{0}^{\text {free }}$ shown in Eq. (7). In particular, taking into account the operator $X$ of Eq. (13), it is crucial to know whether $R_{0}^{\text {free }}$ acts on the left or right side of $X$. The result of the action is the inverse of the sum of the standard frequencies of the corresponding operators,

$R_{0}^{\text {free }}(X)=\frac{X}{\omega_{k_{1}}+\ldots+\omega_{k_{n}}}, \quad(X) R_{0}^{\text {free }}=\frac{X}{\omega_{k_{1}^{\prime}}+\ldots+\omega_{k_{n}^{\prime}}}$.

In the special case where the self-adjoint operator $R_{0}^{\text {free }}$ acts only on the dual state $|0\rangle\langle 0|$, the result is equal to zero [i.e., $R_{0}^{\text {free }}(|0\rangle\langle 0|)=0$ ]. Hence, the perturbation expansion for the zero-temperature equilibrium density matrix around the dual vacuum state is obtained from

$$
\rho_{\text {eq }}=\frac{|\Omega\rangle\langle\Omega|}{\langle\Omega \mid \Omega\rangle},
$$

where $|\Omega\rangle=|0\rangle+|\omega\rangle$ with Eq. (7) for $|\omega\rangle$.

By directly substituting Eqs. (9)-(11) and (16) into Eq. (8), we obtain the following third-order expansion for the correlation function: 


$$
\begin{aligned}
& \tilde{C}_{s}^{A_{2} A_{1}}=\operatorname{tr}\left\{A_{2} \mathcal{R}_{s}^{\text {free }} A_{1}|0\rangle\langle 0|\right. \\
& +\left[A_{2} \mathcal{R}_{s}^{\text {free }} \mathcal{L}^{\text {coll }} \mathcal{R}_{s}^{\text {free }} A_{1}|0\rangle\left\langle 0\left|-A_{2} \mathcal{R}_{s}^{\text {free }} A_{1} R_{0}^{\text {free }} H^{\text {coll }}\right| 0\right\rangle\left\langle 0\left|-A_{2} \mathcal{R}_{s}^{\text {free }} A_{1}\right| 0\right\rangle\langle 0| H^{\text {coll }} R_{0}^{\text {free }}\right] \\
& +\left[A_{2} \mathcal{R}_{s}^{\text {free }} \mathcal{L}^{\text {coll }} \mathcal{R}_{s}^{\text {free }} \mathcal{L}^{\text {coll }} \mathcal{R}_{s}^{\text {free }} A_{1}|0\rangle\left\langle 0\left|-A_{2} \mathcal{R}_{s}^{\text {free }} \mathcal{L}^{\text {coll }} \mathcal{R}_{s}^{\text {free }} A_{1} R_{0}^{\text {free }} H^{\text {coll }}\right| 0\right\rangle\langle 0|\right. \\
& -A_{2} \mathcal{R}_{s}^{\text {free }} \mathcal{L}^{\text {coll }} \mathcal{R}_{s}^{\text {free }} A_{1}|0\rangle\left\langle 0\left|H^{\text {coll }} R_{0}^{\text {free }}+A_{2} \mathcal{R}_{s}^{\text {free }} A_{1} R_{0}^{\text {free }} H^{\text {coll }}\right| 0\right\rangle\langle 0| H^{\text {coll }} R_{0}^{\text {free }} \\
& +A_{2} \mathcal{R}_{s}^{\text {free }} A_{1}\left(R_{0}^{\text {free }} H^{\text {coll }}\right)^{2}|0\rangle\left\langle 0\left|+A_{2} \mathcal{R}_{s}^{\text {free }} A_{1}\right| 0\right\rangle\langle 0|\left(H^{\text {coll }} R_{0}^{\text {free }}\right)^{2} \\
& \left.-A_{2} \mathcal{R}_{s}^{\text {free }} A_{1}|0\rangle\langle 0|\left\langle 0\left|H^{\text {coll }}\left(R_{0}^{\text {free }}\right)^{2} H^{\text {coll }}\right| 0\right\rangle\right] \\
& +\left[A_{2} \mathcal{R}_{s}^{\text {free }} \mathcal{L}^{\text {coll }} \mathcal{R}_{s}^{\text {free }} \mathcal{L}^{\text {coll }} \mathcal{R}_{s}^{\text {free }} \mathcal{L}^{\text {coll }} \mathcal{R}_{s}^{\text {free }} A_{1}|0\rangle\left\langle 0\left|-A_{2} \mathcal{R}_{s}^{\text {free }} \mathcal{L}^{\text {coll }} \mathcal{R}_{s}^{\text {free }} \mathcal{L}^{\text {coll }} \mathcal{R}_{s}^{\text {free }} A_{1} R_{0}^{\text {free }} H^{\text {coll }}\right| 0\right\rangle\langle 0|\right. \\
& -A_{2} \mathcal{R}_{s}^{\text {free }} \mathcal{L}^{\text {coll }} \mathcal{R}_{s}^{\text {free }} \mathcal{L}^{\text {coll }} \mathcal{R}_{s}^{\text {free }} A_{1}|0\rangle\left\langle 0\left|H^{\text {coll }} R_{0}^{\text {free }}+A_{2} \mathcal{R}_{s}^{\text {free }} \mathcal{L}^{\text {coll }} \mathcal{R}_{s}^{\text {free }} A_{1} R_{0}^{\text {free }} H^{\text {coll }}\right| 0\right\rangle\langle 0| H^{\text {coll }} R_{0}^{\text {free }} \\
& +A_{2} \mathcal{R}_{s}^{\text {free }} \mathcal{L}^{\text {coll }} \mathcal{R}_{s}^{\text {free }} A_{1}\left(R_{0}^{\text {free }} H^{\text {coll }}\right)^{2}|0\rangle\left\langle 0\left|+A_{2} \mathcal{R}_{s}^{\text {free }} \mathcal{L}^{\text {coll }} \mathcal{R}_{s}^{\text {free }} A_{1}\right| 0\right\rangle\langle 0|\left(H^{\text {coll }} R_{0}^{\text {free }}\right)^{2} \\
& -A_{2} \mathcal{R}_{s}^{\text {free }} \mathcal{L}^{\text {coll }} \mathcal{R}_{s}^{\text {free }} A_{1}|0\rangle\left\langle 0\left|\left\langle 0\left|H^{\text {coll }}\left(R_{0}^{\text {free }}\right)^{2} H^{\text {coll }}\right| 0\right\rangle-A_{2} \mathcal{R}_{s}^{\text {free }} A_{1} R_{0}^{\text {free }} H^{\text {coll }}\right| 0\right\rangle\langle 0|\left(H^{\text {coll }} R_{0}^{\text {free }}\right)^{2} \\
& -A_{2} \mathcal{R}_{s}^{\text {free }} A_{1}\left(R_{0}^{\text {free }} H^{\text {coll }}\right)^{2}|0\rangle\left\langle 0\left|H^{\text {coll }} R_{0}^{\text {free }}+A_{2} \mathcal{R}_{s}^{\text {free }} A_{1}\right| 0\right\rangle\langle 0|\left\langle 0\left|H^{\text {coll }} R_{0}^{\text {free }}\left(R_{0}^{\text {free }} H^{\text {coll }}\right)^{2}\right| 0\right\rangle \\
& +A_{2} \mathcal{R}_{s}^{\text {free }} A_{1}|0\rangle\langle 0|\left\langle 0\left|\left(H^{\text {coll }} R_{0}^{\text {free }}\right)^{2} R_{0}^{\text {free }} H^{\text {coll }}\right| 0\right\rangle \\
& +A_{2} \mathcal{R}_{s}^{\text {free }} A_{1} R_{0}^{\text {free }} H^{\text {coll }}|0\rangle\langle 0|\left\langle 0\left|H^{\text {coll }}\left(R_{0}^{\text {free }}\right)^{2} H^{\text {coll }}\right| 0\right\rangle \\
& +A_{2} \mathcal{R}_{s}^{\text {free }} A_{1}|0\rangle\langle 0| H^{\text {coll }} R_{0}^{\text {free }}\left\langle 0\left|H^{\text {coll }}\left(R_{0}^{\text {free }}\right)^{2} H^{\text {coll }}\right| 0\right\rangle \\
& \left.\left.-A_{2} \mathcal{R}_{s}^{\text {free }} A_{1}\left(R_{0}^{\text {free }} H^{\text {coll }}\right)^{3}|0\rangle\left\langle 0\left|-A_{2} \mathcal{R}_{s}^{\text {free }} A_{1}\right| 0\right\rangle\langle 0|\left(H^{\text {coll }} R_{0}^{\text {free }}\right)^{3}\right]\right\} \text {. }
\end{aligned}
$$

In deriving Eq. (17), use was made of a symbolic code which is described in detail in a forthcoming publication.

The first term inside the trace on the right-hand side of Eq. (17) corresponds to the zeroth-order contribution, while the second line to the first order. The second-order contribution is displayed in the next four lines. The rest of the terms define the third-order contribution. Overall, Eq. (17) can be decomposed as

$$
\tilde{C}_{s}^{A_{2} A_{1}}=\tilde{C}_{s, 0}^{A_{2} A_{1}}+\tilde{C}_{s, 1}^{A_{2} A_{1}}+\tilde{C}_{s, 2}^{A_{2} A_{1}}+\tilde{C}_{s, 3}^{A_{2} A_{1}}
$$

where the subscripts $0,1,2$, and 3 denote the respective zeroth-, first-, second-, and third-order contributions.

It turns out that one can get the same result [that is, Eq. (17)] through an alternative, more efficient scheme, wherein one utilizes information from already computed lower-order terms to calculate higher-order terms [4]. This scheme is motivated by the stochastic unraveling of the fundamental quantum master equation (see Sec. 1.2.8 of [4]) and leads to a more well-structured and less timeconsuming calculation procedure. It describes interactions between particles as collisions occurring at a certain rate, while treating the dissipative dynamics of the free evolution between collisions rigorously.

\section{THE PROPAGATOR UP TO THIRD ORDER}

We proceed now to the calculation of all terms of Eq. (17) one by one. For completion, we report here not only the expression for the third-order contribution to the propagator of the dissipative, scalar $\varphi^{4}$ theory but also the expressions for the first- and second-order terms which have already been derived in [4].

All the operations involved were computed both by hand and by the symbolic code.

\section{A. Zeroth-order propagator}

The zeroth-order contribution to the two-point correlation function arises from the first line of Eq. (17), with $A_{1}$ and $A_{2}$ given by Eq. (9). The final result after keeping only terms symmetric in $s$ is

$$
\tilde{C}_{s, 0}^{A_{2} A_{1}}=\frac{-\mathrm{i}}{2\left(s^{2}+\omega_{k}^{2}\right)} .
$$

Laplace transforms probe the exponential decay or increase of functions in the direction of positive time. In the case of reversible dynamics, both directions of time are equivalent, and the Laplace transforms are symmetric in the Laplace variable $s$. Irreversible contributions to dynamics lead to predominantly antisymmetric results in $s$. As we are primarily interested in the usual reversible physics and not in small dissipative contributions per se, but only in their regularizing effect on reversible dynamics, we consider only the familiar symmetric part of Laplace transformed correlation functions.

Equation (19) is in agreement with the first term of Eq. (2.31) in [4]; the primary expression for the zerothorder result is the first term in Eq. (2.29) in [4] and one goes to Eq. (19) by neglecting terms antisymmetric in $s$, with the corresponding trace being evaluated by repeated use of the commutation relations for creation and annihilation operators. 


\section{B. First-order propagator}

The first-order term contributing to the propagator is

$$
\tilde{C}_{s, 1}^{A_{2} A_{1}}=\frac{i \lambda^{\prime}}{2\left(s^{2}+\omega_{k}^{2}\right)^{2}},
$$

which is symmetric in $s$ and comes from the second line of Eq. (17). As with the zeroth-order result shown in Sec. III A, Eq. (20) is identical to the second term of Eq. (2.31) in [4]. It is worth mentioning that, to this order, only terms proportional to $\lambda^{\prime}$ [coming from the interaction Hamiltonian, Eq. (4)] contribute.

\section{Second-order propagator}

To compute the second-order terms in the perturbation expansion of Eq. (17), only the four-particle interactions proportional to $\lambda$ [through the interaction Hamiltonian, Eq. (4)] are taken into account, because the terms proportional to $\lambda^{\prime}$ and $\lambda^{\prime \prime}$ contribute to higher-order terms [4]. The final result is obtained after applying once more the SID assumption (i.e., by taking all differences between modified complex frequencies and their conjugate counterparts in the numerators to be zero, since eventually we are interested in the limit of zero dissipation), after symmetrizing with respect to $s$, and after performing the thermodynamic limit (where we pass from $K^{d}$ to $\bar{K}^{d}$ in the sums [4]); it reads

$$
\begin{aligned}
\tilde{C}_{s, 2}^{A_{2} A_{1}}= & -\frac{\lambda^{2}}{48 V^{2}} \frac{\mathrm{i}}{\left(s^{2}+\omega_{k}^{2}\right)^{2}} \\
& \times \sum_{\boldsymbol{k}_{1}, \boldsymbol{k}_{2}, \boldsymbol{k}_{3} \in \bar{K}^{d}} \frac{\delta_{\boldsymbol{k}_{1}+\boldsymbol{k}_{2}+\boldsymbol{k}_{3}, k}}{\omega_{k_{1}} \omega_{k_{2}} \omega_{k_{3}}} \frac{\breve{\omega}_{k_{1}}+\breve{\omega}_{k_{2}}+\breve{\omega}_{k_{3}}}{s^{2}+\left(\breve{\omega}_{k_{1}}+\breve{\omega}_{k_{2}}+\breve{\omega}_{k_{3}}\right)^{2}},
\end{aligned}
$$

originating from the terms between the third and the sixth lines in Eq. (17). The topology of the connected Feynman
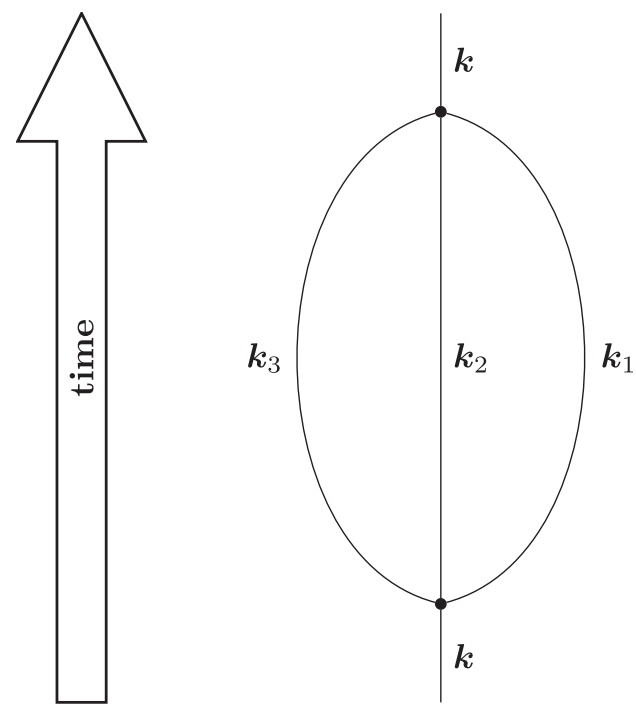

FIG. 1. Topology of the connected Feynman diagram contributing to the propagator to second-order in perturbation theory [4]. diagram depicting the second-order contribution to the propagator is shown in Fig. 1 [4].

\section{Third-order propagator}

The third-order contribution consists of three types of terms: (i) connected-1 (abbreviated as c-1 in the following), (ii) connected-2 (abbreviated as c-2 in the following), and (iii) unconnected terms. A topological representation of the corresponding Feynman diagrams is provided in Figs. 2-4. Additionally, no terms corresponding to tadpole diagrams (i.e., diagrams that contain parts that are attached to the rest of the diagram through a single vertex and are independent
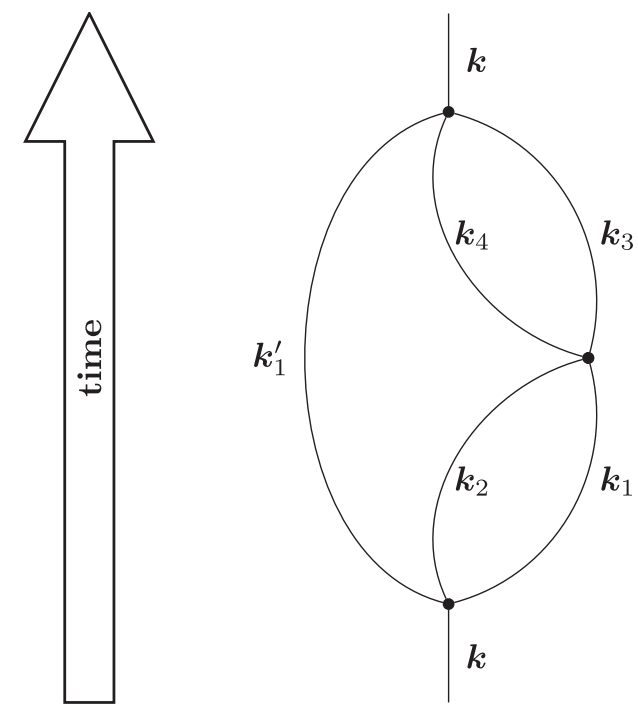

FIG. 2. Topology of the connected-1 Feynman diagram contributing to the propagator to third-order in perturbation theory.
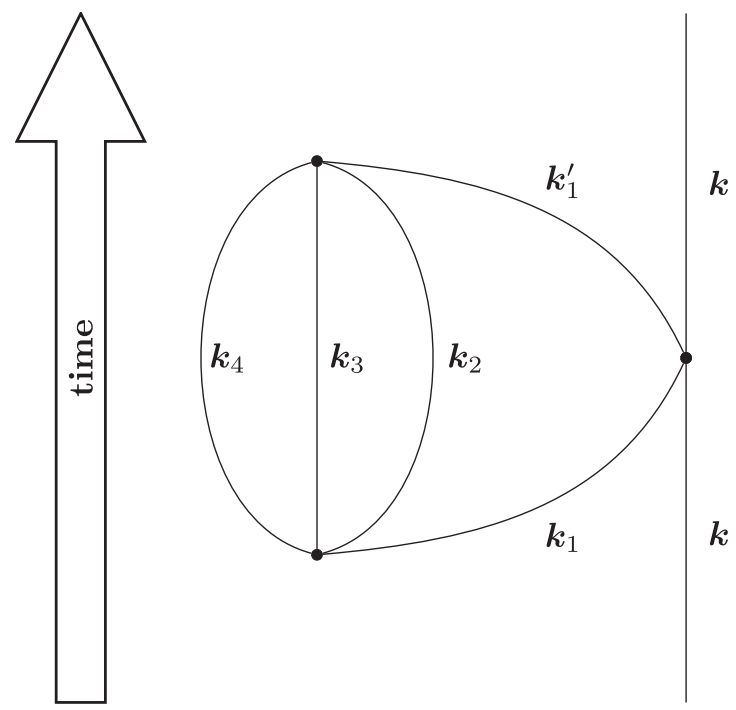

FIG. 3. Topology of the connected-2 Feynman diagram contributing to the propagator in third-order perturbation theory. 


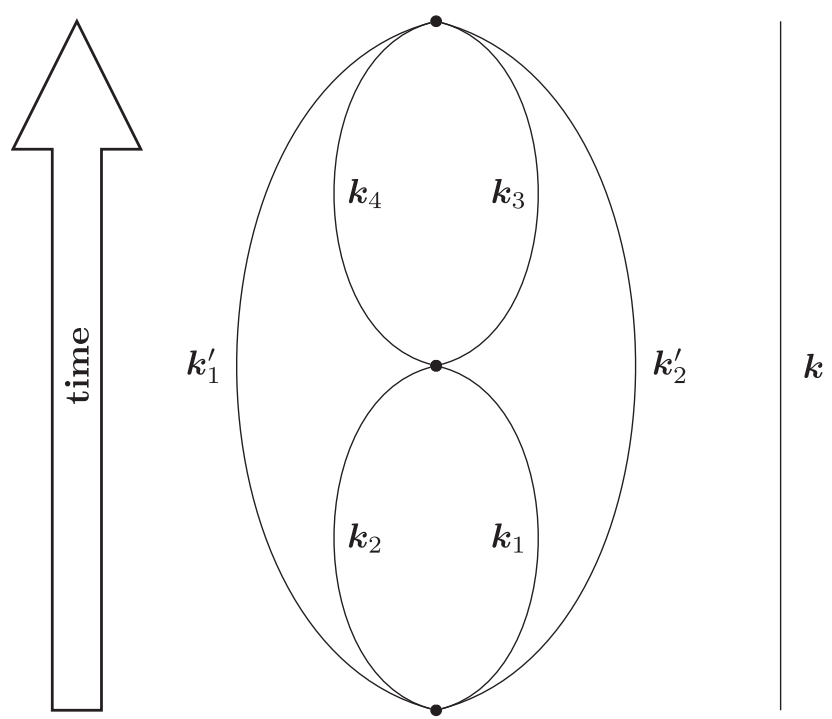

FIG. 4. Topology of the unconnected term in third-order perturbation theory. of the external momentum) are produced due to the repeated use of normal ordering as discussed in [4].

In the corresponding symbolic code, connected- 1 terms involve two Kronecker $\delta$ functions defining a specific relation among the six $d$ vectors of the interacting fields $\left(\boldsymbol{k}_{1}, \boldsymbol{k}_{2}, \boldsymbol{k}_{3}, \boldsymbol{k}_{4}, \boldsymbol{k}_{1}^{\prime}\right)$ and the momentum $\boldsymbol{k}$ of the free particle. The distinguishing feature is that the $k$ index appears only in one of the two Kronecker $\delta$ functions (i.e., $\delta_{\boldsymbol{k}_{1}+\boldsymbol{k}_{2}-\boldsymbol{k}_{3}-\boldsymbol{k}_{4}, \mathbf{0}}$ and $\left.\delta_{\boldsymbol{k}_{1}+\boldsymbol{k}_{2}+\boldsymbol{k}_{1}^{\prime}-\boldsymbol{k}, \mathbf{0}}\right)$. Connected-2 terms are characterized by the complete absence of any Kronecker $\delta$ functions; more specifically, the Kronecker functions in this case are of the form $\delta_{\boldsymbol{k}_{1}+\boldsymbol{k}_{1}^{\prime}, \mathbf{0}}$ and $\delta_{\boldsymbol{k}_{1}+\boldsymbol{k}_{2}+\boldsymbol{k}_{3}+\boldsymbol{k}_{4}, \mathbf{0}}$ independent of the external momentum $\boldsymbol{k}$. Unconnected contributions to the propagator contain two Kronecker $\delta$ functions, none of which includes the momentum index $k$ of the propagating particle.

The final result for the third-order contribution to the propagator is obtained by (a) keeping all the terms which are symmetric with respect to the $s$ variable, (b) applying the SID assumption, and (c) symmetrizing the intermediate result with respect to the pairs of indices $\left(k_{1}, k_{2}\right)$ and $\left(k_{3}, k_{4}\right)$. The resulting expression for the connected-1 term is

$$
\begin{aligned}
& \tilde{C}_{s, 3(\mathrm{c}-1)}^{A_{2} A_{1}}=\frac{\lambda^{3}}{128 V^{3}} \frac{\mathrm{i}}{\left(s^{2}+\omega_{k}^{2}\right)^{2}} \sum_{\boldsymbol{k}_{1}, \boldsymbol{k}_{2}, \boldsymbol{k}_{3}, \boldsymbol{k}_{4}, \boldsymbol{k}_{1}^{\prime} \in \bar{K}^{d}} \frac{\delta_{\boldsymbol{k}_{1}+\boldsymbol{k}_{2}-\boldsymbol{k}_{3}-\boldsymbol{k}_{4}, \boldsymbol{0}} \delta_{\boldsymbol{k}_{1}+\boldsymbol{k}_{2}+\boldsymbol{k}_{1}^{\prime}-\boldsymbol{k}, \mathbf{0}}}{\omega_{k_{1}} \omega_{k_{2}} \omega_{k_{3}} \omega_{k_{4}} \omega_{k_{1}^{\prime}}} \\
& \times\left[\frac{1}{\breve{\omega}_{k_{1}}+\breve{\omega}_{k_{2}}-\breve{\omega}_{k_{3}}-\breve{\omega}_{k_{4}}}\left(\frac{\breve{\omega}_{k_{3}}+\breve{\omega}_{k_{4}}+\breve{\omega}_{k_{1}^{\prime}}}{s^{2}+\left(\breve{\omega}_{k_{3}}+\breve{\omega}_{k_{4}}+\breve{\omega}_{k_{1}^{\prime}}\right)^{2}}-\frac{\breve{\omega}_{k_{1}}+\breve{\omega}_{k_{2}}+\breve{\omega}_{k_{1}^{\prime}}}{s^{2}+\left(\breve{\omega}_{k_{1}}+\breve{\omega}_{k_{2}}+\breve{\omega}_{k_{1}^{\prime}}\right)^{2}}\right)\right.
\end{aligned}
$$

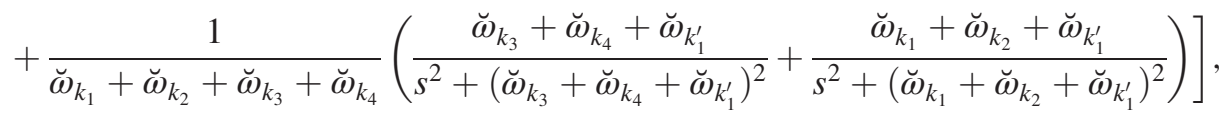

and for the connected-2 term

$$
\tilde{C}_{s, 3(\mathrm{c}-2)}^{A_{2} A_{1}}=\frac{\lambda^{3}}{192 V^{3}} \frac{\mathrm{i}}{\left(s^{2}+\omega_{k}^{2}\right)^{2}} \sum_{k_{1}, k_{2}, k_{3}, k_{4} \in \bar{K}^{d}} \frac{\delta_{k_{1}-k_{2}-k_{3}-k_{4}, 0}}{\omega_{k_{1}}^{2} \omega_{k_{2}} \omega_{k_{3}} \omega_{k_{4}}} \frac{\left(2 \breve{\omega}_{k_{1}}+\breve{\omega}_{k_{2}}+\breve{\omega}_{k_{3}}+\breve{\omega}_{k_{4}}\right)}{\left.2 \breve{\omega}_{k_{1}}+\breve{\omega}_{k_{2}}+\breve{\omega}_{k_{3}}+\breve{\omega}_{k_{4}}\right)^{2}} .
$$

Overall, summing up the terms shown in Eqs. (19), (20), (21), (22), and (23), we arrive at the following compact expression for the propagator up to third order in $\lambda$ (with $\tilde{C}_{s}^{A_{2} A_{1}} \equiv \tilde{\Delta}_{s k}$ ):

$$
\begin{aligned}
& \tilde{\Delta}_{s k}=\frac{-\mathrm{i}}{2\left(s^{2}+\omega_{k}^{2}\right)}+\frac{\mathrm{i} \lambda^{\prime}}{2\left(s^{2}+\omega_{k}^{2}\right)^{2}}-\frac{\lambda^{2}}{48 V^{2}} \frac{\mathrm{i}}{\left(s^{2}+\omega_{k}^{2}\right)^{2}} \sum_{k_{1}, k_{2}, k_{3} \in \bar{K}^{d}} \frac{\delta_{k_{1}+k_{2}+k_{3}, k}}{\omega_{k_{1}} \omega_{k_{2}} \omega_{k_{3}}} \frac{\breve{\omega}_{k_{1}}+\breve{\omega}_{k_{2}}+\breve{\omega}_{k_{3}}}{s^{2}+\left(\breve{\omega}_{k_{1}}+\breve{\omega}_{k_{2}}+\breve{\omega}_{k_{3}}\right)^{2}} \\
& +\frac{\lambda^{3}}{128 V^{3}} \frac{\mathrm{i}}{\left(s^{2}+\omega_{k}^{2}\right)^{2}} \sum_{\boldsymbol{k}_{1}, \boldsymbol{k}_{2}, \boldsymbol{k}_{3}, \boldsymbol{k}_{4}, \boldsymbol{k}_{1}^{\prime} \in \bar{K}^{d}} \frac{\delta_{\boldsymbol{k}_{1}+\boldsymbol{k}_{2}-\boldsymbol{k}_{3}-\boldsymbol{k}_{4}, \boldsymbol{0}} \delta_{\boldsymbol{k}_{1}+\boldsymbol{k}_{2}+\boldsymbol{k}_{1}^{\prime}, \boldsymbol{k}}}{\omega_{k_{1}} \omega_{k_{2}} \omega_{k_{3}} \omega_{k_{4}} \omega_{k_{1}^{\prime}}} \\
& \times\left[\frac{1}{\breve{\omega}_{k_{1}}+\breve{\omega}_{k_{2}}-\breve{\omega}_{k_{3}}-\breve{\omega}_{k_{4}}}\left(\frac{\breve{\omega}_{k_{3}}+\breve{\omega}_{k_{4}}+\breve{\omega}_{k_{1}^{\prime}}}{s^{2}+\left(\breve{\omega}_{k_{3}}+\breve{\omega}_{k_{4}}+\breve{\omega}_{k_{1}^{\prime}}\right)^{2}}-\frac{\breve{\omega}_{k_{1}}+\breve{\omega}_{k_{2}}+\breve{\omega}_{k_{1}^{\prime}}}{s^{2}+\left(\breve{\omega}_{k_{1}}+\breve{\omega}_{k_{2}}+\breve{\omega}_{k_{1}^{\prime}}\right)^{2}}\right)\right. \\
& \left.+\frac{1}{\breve{\omega}_{k_{1}}+\breve{\omega}_{k_{2}}+\breve{\omega}_{k_{3}}+\breve{\omega}_{k_{4}}}\left(\frac{\breve{\omega}_{k_{3}}+\breve{\omega}_{k_{4}}+\breve{\omega}_{k_{1}^{\prime}}}{s^{2}+\left(\breve{\omega}_{k_{3}}+\breve{\omega}_{k_{4}}+\breve{\omega}_{k_{1}^{\prime}}\right)^{2}}+\frac{\breve{\omega}_{k_{1}}+\breve{\omega}_{k_{2}}+\breve{\omega}_{k_{1}^{\prime}}}{s^{2}+\left(\breve{\omega}_{k_{1}}+\breve{\omega}_{k_{2}}+\breve{\omega}_{k_{1}^{\prime}}\right)^{2}}\right)\right] \\
& +\frac{\lambda^{3}}{192 V^{3}} \frac{i}{\left(s^{2}+\omega_{k}^{2}\right)^{2}} \sum_{k_{1}, k_{2}, k_{3}, k_{4} \in \bar{K}^{d}} \frac{\delta_{k_{1}-k_{2}-k_{3}-k_{4}, \mathbf{0}}}{\omega_{k_{1}}^{2} \omega_{k_{2}} \omega_{k_{3}} \omega_{k_{4}}} \frac{\left(2 \breve{\omega}_{k_{1}}+\breve{\omega}_{k_{2}}+\breve{\omega}_{k_{3}}+\breve{\omega}_{k_{1}}+\breve{\omega}_{k_{2}}+\breve{\omega}_{k_{3}}+\breve{\omega}_{k_{4}}\right)^{2}}{2 .}
\end{aligned}
$$


Equation (24) was obtained through our symbolic code and by hand (the latter might not be easy for higher-order contributions), and it is the key result of this work. In the next sections of the paper, we discuss (i) the reduced form of Eq. (24) in the limit of vanishing dissipation and the equivalence with Lagrangian quantum field theory, (ii) the regularization of Eq. (24) so that divergences are avoided, and (iii) the critical behavior of the theory.

\section{LIMIT OF VANISHING DISSIPATION: EQUIVALENCE WITH LAGRANGIAN QUANTUM FIELD THEORY}

Of interest is how the previous expressions for the connected contributions up to third order reduce in the limit of vanishing friction parameter, $\gamma \rightarrow 0$. This is important because it allows us to make the connection with the expressions for the propagator evaluated by using the $D$-vector formalism of the Lagrangian approach.

Equations (21)-(23) in the limit of vanishing dissipation (by replacing $\breve{\omega}_{k_{j}}$ with $\omega_{k_{j}}$ ) must coincide with those obtained from the Lagrangian approach in $D=d+1$ dimensions ( $d$ denotes the number of space dimensions and $D$ the number of space + time dimensions) with the use of $D$ vectors. To prove this, we can bring our result in integral form and then go from $\tilde{C}_{s}^{A_{2} A_{1}}$ to $\tilde{C}_{\mathrm{i} \omega}^{A_{2} A_{1}}$ and finally to $\mathrm{i} C_{\omega}^{A_{2} A_{1}}$. This is achieved as follows: (a) We pass from sums to integrals (corresponding to the limit of infinite volume) by using the rule shown in Eq. (2.41) of [4],

$$
\frac{1}{V} \sum_{k \in \bar{K}^{d}} \rightarrow \frac{1}{(2 \pi)^{d}} \int \mathrm{d}^{d} k
$$

and from the Kronecker $\delta$ symbol to the Dirac $\delta$ function. (b) Then, we pass from Laplace to Fourier transforms (i.e., from positive $s$ to imaginary $\pm \omega$ ) by setting $s \rightarrow \pm \mathrm{i} \omega$. (c) Afterwards, we observe the symmetry property $\tilde{\Delta}_{s k}=$ $\tilde{\Delta}_{s-k}$ of the second- and third-order contributions and use [4]

$$
\mathrm{i} C_{\omega}^{A_{2} A_{1}}=\tilde{C}_{\mathrm{i} \omega}^{A_{2} A_{1}}+\tilde{C}_{-\mathrm{i} \omega}^{A_{1} A_{2}},
$$

and

$$
\mathrm{i} \Delta_{\omega k}=\tilde{\Delta}_{\mathrm{i} \omega k}+\tilde{\Delta}_{-\mathrm{i} \omega-k} .
$$

Note that in the passage from $\tilde{C}_{\mathrm{i} \omega k}$ to $\mathrm{i} C_{\omega k}$ there is an extra multiplying factor of 2 due to Eq. (26b) (i.e., $\mathrm{i} C_{\omega}^{A_{2} A_{1}}=2 \tilde{C}_{\mathrm{i} \omega}^{A_{2} A_{1}}$ ).

On the other hand, in the Lagrangian approach, we obtain the contributions to the propagator in $D$ dimensions with the help of the Feynman diagrams shown in Figs. 1-3 $[2,13]$. The $D$ components of a space-time vector $\underline{k}_{j}$ are written as $\underline{k}_{j}=\left(\kappa_{j}, \boldsymbol{k}_{j}\right)$, where $\kappa_{j}$ is real and $\boldsymbol{k}_{j}$ has $d$ components. Similarly, we use $\underline{k}=(\omega, \boldsymbol{k})$. It is significant to mention that throughout this work we use the Minkowski metric $\eta^{\mu \nu}=\eta_{\mu \nu}$ with signature $(-,+,+,+)$, that is, $\eta^{00}=\eta_{00}=-1$ (see Appendix $\mathrm{E}$ of [13]). Then, we integrate out the zero component of the $D$ vectors, reducing to $d$-dimensional expressions. The calculation proceeds by replacing the factors contained in the $D$-dimensional $\delta$ functions by their one-dimensional Fourier representation and then performing the factorized integrations over momenta by means of Cauchy's integral formula. Therefore, one can perform the integration resulting from the Fourier representation of the $\delta$ functions (see also the identity shown in Eq. (2.57) of [4] regarding the secondorder term).

By doing these calculations, we arrive at the following expressions:

$$
\begin{aligned}
& C_{\omega, 2}^{d-\mathrm{vec}}=\frac{-\mathrm{i}}{\left(\omega^{2}-\omega_{k}^{2}+\mathrm{i} \varepsilon\right)^{2}} \frac{1}{2^{2} C_{\mathrm{sf}}^{(2)}} \frac{\lambda^{2}}{(2 \pi)^{2 d}} \int \frac{\mathrm{d}^{d} k_{1} \mathrm{~d}^{d} k_{2} \mathrm{~d}^{d} k_{3}}{\omega_{k_{1}} \omega_{k_{2}} \omega_{k_{3}}} \delta\left(\boldsymbol{k}_{1}+\boldsymbol{k}_{2}+\boldsymbol{k}_{3}-\boldsymbol{k}\right) \frac{\omega_{k_{1}}+\omega_{k_{2}}+\omega_{k_{3}}}{\left(\omega_{k_{1}}+\omega_{k_{2}}+\omega_{k_{3}}\right)^{2}-\omega^{2}}, \\
& C_{\omega, 3(\mathrm{c}-1)}^{d \text {-vec }}= \frac{\mathrm{i}}{\left(\omega^{2}-\omega_{k}^{2}+\mathrm{i} \varepsilon\right)^{2}} \frac{1}{2^{4} C_{\mathrm{sf}}^{(3), \mathrm{c}-1}} \frac{\lambda^{3}}{(2 \pi)^{3 d}} \int \mathrm{d}^{d} k_{1} \mathrm{~d}^{d} k_{2} \mathrm{~d}^{d} k_{3} \mathrm{~d}^{d} k_{4} \mathrm{~d}^{d} k_{1}^{\prime} \frac{\delta\left(\boldsymbol{k}_{1}+\boldsymbol{k}_{2}+\boldsymbol{k}_{1}^{\prime}-\boldsymbol{k}\right) \delta\left(\boldsymbol{k}_{1}+\boldsymbol{k}_{2}-\boldsymbol{k}_{3}-\boldsymbol{k}_{4}\right)}{\omega_{k_{1}} \omega_{k_{2}} \omega_{k_{3}} \omega_{k_{4}} \omega_{k_{1}^{\prime}}} \\
& \times\left[\frac{1}{\omega_{k_{1}}+\omega_{k_{2}}-\omega_{k_{3}}-\omega_{k_{4}}}\left(\frac{\omega_{k_{3}}+\omega_{k_{4}}+\omega_{k_{1}^{\prime}}}{\left(\omega_{k_{3}}+\omega_{k_{4}}+\omega_{k_{1}^{\prime}}\right)^{2}-\omega^{2}}-\frac{\omega_{k_{1}}+\omega_{k_{2}}+\omega_{k_{1}^{\prime}}}{\left(\omega_{k_{1}}+\omega_{k_{2}}+\omega_{k_{1}^{\prime}}\right)^{2}-\omega^{2}}\right)\right. \\
&+\frac{1}{\omega_{k_{1}}+\omega_{k_{2}}+\omega_{k_{3}}+\omega_{k_{4}}}\left(\frac{\omega_{k_{3}}+\omega_{k_{4}}+\omega_{k_{1}^{\prime}}}{\left(\omega_{k_{3}}+\omega_{k_{4}}+\omega_{k_{1}^{\prime}}\right)^{2}-\omega^{2}}+\frac{\omega_{k_{1}}+\omega_{k_{2}}+\omega_{k_{1}^{\prime}}}{\left.\left.\left(\omega_{k_{1}}+\omega_{k_{2}}+\omega_{k_{1}^{\prime}}\right)^{2}-\omega^{2}\right)\right],}\right.
\end{aligned}
$$

and 


$$
\begin{aligned}
C_{\omega, 3(\mathrm{c}-2)}^{d-\mathrm{ec}}= & \frac{-\mathrm{i}}{\left(\omega^{2}-\omega_{k}^{2}+\mathrm{i} \varepsilon\right)^{2}} \frac{1}{2^{3} C_{\mathrm{sf}}^{(3), \mathrm{c}-2}} \frac{\lambda^{3}}{(2 \pi)^{3 d}} \int \frac{\mathrm{d}^{d} k_{1} \mathrm{~d}^{d} k_{2} \mathrm{~d}^{d} k_{3} \mathrm{~d}^{d} k_{4}}{\omega_{k_{1}}^{2} \omega_{k_{2}} \omega_{k_{3}} \omega_{k_{4}}} \delta\left(\boldsymbol{k}_{1}-\boldsymbol{k}_{2}-\boldsymbol{k}_{3}-\boldsymbol{k}_{4}\right) \\
& \times\left\{\frac{1}{\omega_{k_{1}}^{2}-\left(\omega_{k_{2}}+\omega_{k_{3}}+\omega_{k_{4}}\right)^{2}}\left[\frac{\left[\left(\omega_{k_{2}}+\omega_{k_{3}}+\omega_{k_{4}}\right)-\omega_{k_{1}}\right]\left(2 \omega_{k_{1}}+\omega_{k_{2}}+\omega_{k_{3}}+\omega_{k_{4}}\right)}{2 \omega_{k_{1}}\left(\omega_{k_{1}}+\omega_{k_{2}}+\omega_{k_{3}}+\omega_{k_{4}}\right)}\right]\right\} \\
= & \frac{\mathrm{i}}{\left(\omega^{2}-\omega_{k}^{2}+\mathrm{i} \varepsilon\right)^{2}} \frac{1}{2^{3} C_{\mathrm{sf}}^{(3), \mathrm{c}-2}} \frac{\lambda^{3}}{(2 \pi)^{3 d}} \\
& \times \int \frac{\mathrm{d}^{d} k_{1} \mathrm{~d}^{d} k_{2} \mathrm{~d}^{d} k_{3} \mathrm{~d}^{d} k_{4}}{\omega_{k_{1}}^{2} \omega_{k_{2}} \omega_{k_{3}} \omega_{k_{4}}} \delta\left(\boldsymbol{k}_{1}-\boldsymbol{k}_{2}-\boldsymbol{k}_{3}-\boldsymbol{k}_{4}\right) \frac{\left(2 \omega_{k_{1}}+\omega_{k_{2}}+\omega_{k_{3}}+\omega_{k_{4}}\right)}{2 \omega_{k_{1}}\left(\omega_{k_{1}}+\omega_{k_{2}}+\omega_{k_{3}}+\omega_{k_{4}}\right)^{2}},
\end{aligned}
$$

where $C_{\mathrm{sf}}^{(2)}=6, C_{\mathrm{sf}}^{(3), \mathrm{c}-1}=4$ and $C_{\mathrm{sf}}^{(3), \mathrm{c}-2}=12$ denote the symmetry factors of the second-order, third-order connected-1, and third-order connected-2 diagrams, respectively \{e.g., see Eq. (4.27) of [14] or Eq. (11) of [15] or p. 93 of [2]\}. The above expressions, Eqs. (27)-(29), coincide with the nondissipative version of Eqs. (21)-(23) by also making use of Eqs. (25) and (26).

This fundamental result demonstrates the complete equivalence of the dissipative approach (in the limit of zero dissipation) to the Lagrangian method.

\section{REGULARIZATION}

As discussed in [4], the parameters $\lambda^{\prime}$ and $Z$ are not unique, and their values can be chosen in such a way so as to regularize the expression for the propagator obtained when we apply the limits of (a) infinite system volume $V$ and (b) vanishing friction parameter $\gamma$. A natural condition to impose is

$\mathrm{i}\left(s^{2}+\breve{\omega}_{k}^{2}\right)^{2} Z \tilde{\Delta}_{s k}=\mathrm{i}\left(s^{2}+\breve{\omega}_{k}^{2}\right)^{2} \tilde{\Delta}_{s \boldsymbol{k}}^{\text {free }}, \quad$ for $\boldsymbol{k}=\mathbf{0}$,

where, according to Eq. (19), $\tilde{\Delta}_{s k}^{\text {free }}=(-\mathrm{i}) /\left[2\left(s^{2}+\omega_{k}^{2}\right)\right]$. By substituting Eq. (24) into Eq. (30), expanding the lefthand side up to second order in $s$ using the expansion

$$
\frac{\breve{\omega}}{s^{2}+\breve{\omega}^{2}}=\frac{1}{\breve{\omega}} \sum_{n=0}^{\infty}\left(-\frac{s^{2}}{\breve{\omega}^{2}}\right)^{n},
$$

and matching terms order by order with the right-hand side, we obtain the following expressions in the context of the SID assumption:

$$
\begin{aligned}
Z= & 1+\frac{\lambda^{2}}{24 V^{2}} \sum_{k_{1}, k_{2}, k_{3} \in \bar{K}^{d}} \frac{\delta_{\boldsymbol{k}_{1}+\boldsymbol{k}_{2}+\boldsymbol{k}_{3}, \mathbf{0}}}{\omega_{k_{1}} \omega_{k_{2}} \omega_{k_{3}}} \frac{1}{\left(\breve{\omega}_{k_{1}}+\breve{\omega}_{k_{2}}+\breve{\omega}_{k_{3}}\right)^{3}}-\frac{\lambda^{3}}{64 V^{3}} \sum_{\boldsymbol{k}_{1}, \boldsymbol{k}_{2}, k_{3}, k_{4}, k_{1}^{\prime} \in \bar{K}^{d}} \frac{\delta_{\boldsymbol{k}_{1}+\boldsymbol{k}_{2}-\boldsymbol{k}_{3}-\boldsymbol{k}_{4}, \mathbf{0}} \delta_{\boldsymbol{k}_{1}+\boldsymbol{k}_{2}+\boldsymbol{k}_{1}^{\prime}, \mathbf{0}}}{\omega_{k_{1}} \omega_{k_{2}} \omega_{k_{3}} \omega_{k_{4}} \omega_{k_{1}^{\prime}}} \\
& \times\left[\frac{1}{\breve{\omega}_{k_{1}}+\breve{\omega}_{k_{2}}-\breve{\omega}_{k_{3}}-\breve{\omega}_{k_{4}}}\left(\frac{1}{\left(\breve{\omega}_{k_{3}}+\breve{\omega}_{k_{4}}+\breve{\omega}_{k_{1}^{\prime}}\right)^{3}}-\frac{1}{\left(\breve{\omega}_{k_{1}}+\breve{\omega}_{k_{2}}+\breve{\omega}_{k_{1}^{\prime}}\right)^{3}}\right)\right. \\
& \left.+\frac{1}{\breve{\omega}_{k_{1}}+\breve{\omega}_{k_{2}}+\breve{\omega}_{k_{3}}+\breve{\omega}_{k_{4}}}\left(\frac{1}{\left(\breve{\omega}_{k_{3}}+\breve{\omega}_{k_{4}}+\breve{\omega}_{k_{1}^{\prime}}\right)^{3}}+\frac{1}{\left(\breve{\omega}_{k_{1}}+\breve{\omega}_{k_{2}}+\breve{\omega}_{k_{1}^{\prime}}\right)^{3}}\right)\right]
\end{aligned}
$$

and

$$
\begin{aligned}
& \lambda^{\prime}=\frac{\lambda^{2}}{24 V^{2}} \sum_{\boldsymbol{k}_{1}, \boldsymbol{k}_{2}, \boldsymbol{k}_{3} \in \bar{K}^{d}} \frac{\delta_{\boldsymbol{k}_{1}+\boldsymbol{k}_{2}+\boldsymbol{k}_{3}, \boldsymbol{0}}}{\omega_{k_{1}} \omega_{k_{2}} \omega_{k_{3}}}\left[\frac{1}{\breve{\omega}_{k_{1}}+\breve{\omega}_{k_{2}}+\breve{\omega}_{k_{3}}}+\frac{m^{2}}{\left(\breve{\omega}_{k_{1}}+\breve{\omega}_{k_{2}}+\breve{\omega}_{k_{3}}\right)^{3}}\right]-\frac{\lambda^{3}}{64 V^{3}} \sum_{\boldsymbol{k}_{1}, \boldsymbol{k}_{2}, \boldsymbol{k}_{3}, \boldsymbol{k}_{4}, \boldsymbol{k}_{1}^{\prime} \in \bar{K}^{d}} \frac{\delta_{\boldsymbol{k}_{1}+\boldsymbol{k}_{2}-\boldsymbol{k}_{3}-\boldsymbol{k}_{4}, \mathbf{0}} \delta_{\boldsymbol{k}_{1}+\boldsymbol{k}_{2}+\boldsymbol{k}_{1}^{\prime}, \mathbf{0}}}{\omega_{k_{1}} \omega_{k_{2}} \omega_{k_{3}} \omega_{k_{4}} \omega_{k_{1}^{\prime}}} \\
& \times\left[\frac{1}{\breve{\omega}_{k_{1}}+\breve{\omega}_{k_{2}}-\breve{\omega}_{k_{3}}-\breve{\omega}_{k_{4}}}\left(\frac{1}{\breve{\omega}_{k_{3}}+\breve{\omega}_{k_{4}}+\breve{\omega}_{k_{1}^{\prime}}}-\frac{1}{\breve{\omega}_{k_{1}}+\breve{\omega}_{k_{2}}+\breve{\omega}_{k_{1}^{\prime}}}\right)\right. \\
& +\frac{1}{\breve{\omega}_{k_{1}}+\breve{\omega}_{k_{2}}+\breve{\omega}_{k_{3}}+\breve{\omega}_{k_{4}}}\left(\frac{1}{\breve{\omega}_{k_{3}}+\breve{\omega}_{k_{4}}+\breve{\omega}_{k_{1}^{\prime}}}+\frac{1}{\breve{\omega}_{k_{1}}+\breve{\omega}_{k_{2}}+\breve{\omega}_{k_{1}^{\prime}}}\right) \\
& +\frac{m^{2}}{\breve{\omega}_{k_{1}}+\breve{\omega}_{k_{2}}-\breve{\omega}_{k_{3}}-\breve{\omega}_{k_{4}}}\left(\frac{1}{\left(\breve{\omega}_{k_{3}}+\breve{\omega}_{k_{4}}+\breve{\omega}_{k_{1}^{\prime}}\right)^{3}}-\frac{1}{\left(\breve{\omega}_{k_{1}}+\breve{\omega}_{k_{2}}+\breve{\omega}_{k_{1}^{\prime}}\right)^{3}}\right) \\
& \left.+\frac{m^{2}}{\breve{\omega}_{k_{1}}+\breve{\omega}_{k_{2}}+\breve{\omega}_{k_{3}}+\breve{\omega}_{k_{4}}}\left(\frac{1}{\left(\breve{\omega}_{k_{3}}+\breve{\omega}_{k_{4}}+\breve{\omega}_{k_{1}^{\prime}}\right)^{3}}+\frac{1}{\left(\breve{\omega}_{k_{1}}+\breve{\omega}_{k_{2}}+\breve{\omega}_{k_{1}^{\prime}}\right)^{3}}\right)\right] \\
& -\frac{\lambda^{3}}{96 V^{3}} \sum_{\boldsymbol{k}_{1}, k_{2}, k_{3}, k_{4} \in \bar{K}^{d}} \frac{\delta_{k_{1}-k_{2}-k_{3}-k_{4}, 0}}{\omega_{k_{1}}^{2} \omega_{k_{2}} \omega_{k_{3}} \omega_{k_{4}}} \frac{\left(2 \breve{\omega}_{k_{1}}+\breve{\omega}_{k_{2}}+\breve{\omega}_{k_{3}}+\breve{\omega}_{k_{4}}\right)}{\left.2 \breve{\omega}_{k_{2}}+\breve{\omega}_{k_{3}}+\breve{\omega}_{k_{4}}\right)^{2}} \text {. }
\end{aligned}
$$


From Eqs. (32) and (33), one can make the following remarks regarding third-order contributions: (a) The connected-1 terms are included in both $Z$ and $\lambda^{\prime}$ (the terms with the factor 1/64). (b) The connected-2 terms contribute only to $\lambda^{\prime}$ (the term with the factor 1/96), since they do not depend on the external momentum $\boldsymbol{k}$ of the free particle.

The above are the values of $Z$ and $\lambda^{\prime}$ for which we expect a well-defined correlation function in the limit of vanishing friction, for all $s$ and $\boldsymbol{k}$. In order to obtain the regularized expression for the propagator, we do the following (see also the Appendix): (i) Using Eqs. (32) and (33) for $\gamma=0$, we obtain the expression for $\left(s^{2}+\omega_{k}^{2}\right)^{2}\left(Z \tilde{\Delta}_{s k}-\tilde{\Delta}_{s k}^{\text {free }}\right)$ in the limit of vanishing friction. A nice point to note here is that the contribution from the connected-2 diagram [the last two lines in Eq. (24)] vanishes. (ii) Afterwards, we analyze the convergence characteristics of the resulting expression, using the expansion shown in Eq. (31). In the consequent expression, Eq. (A1), there are terms proportional to $\left(\omega_{k_{a}}+\omega_{k_{b}}+\right.$ $\left.\omega_{k_{c}}\right)^{-1}$ which lead to a power-law divergence for $d>2$, as well as terms proportional to $\left(\omega_{k_{a}}+\omega_{k_{b}}+\omega_{k_{c}}\right)^{-3}$ leading to a logarithmic divergence for $d=3$. In order to avoid divergent results, the sums containing the above problematic terms, Eq. (A2), must remain finite for an infinitely large lattice $\bar{K}^{3}$ of momentum vectors in $d=3$ dimensions. This is ensured through a numerical calculation. (iii) Subsequently, we pass from sums to integrals (corresponding to the limit of infinite volume) using the rule shown in Eq. (25) and from the Kronecker $\delta$ symbol to the Dirac $\delta$ function with the help of Eq. (26b). (iv) Furthermore, we would like the resulting expression to be covariant and hence to depend only on $\left(\omega^{2}-k^{2}\right)$. In [4], it was shown that this is indeed the case for the second-order propagator. In Sec. IV, we discussed the equivalence between the $D$-dimensional and the $d$-dimensional (by integrating the zero component) expressions obtained from the Lagrangian approach, demonstrating also the covariance of the third-order terms. This allows us to rewrite the resulting expression for $\boldsymbol{k}=\mathbf{0}$, in a different form, Eq. (A3), which can be further simplified to

$$
\begin{aligned}
\left(Z \Delta_{\omega k}-\Delta_{\omega k}^{\text {free }}\right)= & -\frac{\lambda^{2}}{24(2 \pi)^{2 d}} \frac{\left(\omega^{2}-k^{2}\right)^{2}}{\left(\omega^{2}-\omega_{k}^{2}\right)^{2}} \int \frac{\mathrm{d}^{d} k_{1} \mathrm{~d}^{d} k_{2} \mathrm{~d}^{d} k_{3}}{\omega_{k_{1}} \omega_{k_{2}} \omega_{k_{3}}} \delta\left(\boldsymbol{k}_{1}+\boldsymbol{k}_{2}+\boldsymbol{k}_{3}\right) \frac{1}{\left(\omega_{k_{1}}+\omega_{k_{2}}+\omega_{k_{3}}\right)^{3}} \frac{1}{\left(\omega_{k_{1}}+\omega_{k_{2}}+\omega_{k_{3}}\right)^{2}-\left(\omega^{2}-k^{2}\right)} \\
& +\frac{\lambda^{3}}{64(2 \pi)^{3 d}} \frac{2\left(\omega^{2}-k^{2}\right)^{2}}{\left(\omega^{2}-\omega_{k}^{2}\right)^{2}} \int \frac{\mathrm{d}^{d} k_{1} \mathrm{~d}^{d} k_{2} \mathrm{~d}^{d} k_{3} \mathrm{~d}^{d} k_{4} \mathrm{~d}^{d} k_{1}^{\prime}}{\omega_{k_{1}} \omega_{k_{2}} \omega_{k_{3}} \omega_{k_{4}} \omega_{k_{1}^{\prime}}} \delta\left(\boldsymbol{k}_{1}+\boldsymbol{k}_{2}-\boldsymbol{k}_{3}-\boldsymbol{k}_{4}\right) \delta\left(\boldsymbol{k}_{1}+\boldsymbol{k}_{2}+\boldsymbol{k}_{1}^{\prime}\right) \\
& \times \frac{1}{\left(\omega_{k_{1}}+\omega_{k_{2}}-\omega_{k_{3}}-\omega_{k_{4}}\right)\left(\omega_{k_{1}}+\omega_{k_{2}}+\omega_{k_{3}}+\omega_{k_{4}}\right)}\left[\frac{\omega_{k_{1}}+\omega_{k_{2}}}{\left(\omega_{k_{3}}+\omega_{k_{4}}+\omega_{k_{1}^{\prime}}\right)^{3}\left[\left(\omega_{k_{3}}+\omega_{k_{4}}+\omega_{k_{1}^{\prime}}\right)^{2}-\left(\omega^{2}-k^{2}\right)\right]}\right. \\
& \left.-\frac{\omega_{k_{3}}+\omega_{k_{4}}}{\left(\omega_{k_{1}}+\omega_{k_{2}}+\omega_{k_{1}^{\prime}}\right)^{3}\left[\left(\omega_{k_{1}}+\omega_{k_{2}}+\omega_{k_{1}^{\prime}}\right)^{2}-\left(\omega^{2}-k^{2}\right)\right]}\right]
\end{aligned}
$$

which is the regularized result of our dissipative approach at zero friction. A direct power counting in Eq. (34) proves that all terms are nicely convergent for $d \leq 3$, thus producing a finite result, which is what we wanted to achieve.

In an alternative regularization scheme, the so-called "on shell renormalization", one can expand not with respect to $\omega^{2}-k^{2}$ but with respect to $\omega^{2}-k^{2}-m^{2}$ [when going from Eq. (A3) to Eq. (34)]. This scheme indicates that one stays near the physical relation between the frequency $\omega$ and the wave vector $\boldsymbol{k}$ for a massive particle with mass $m$. In this scheme, the final expression for the second- and third-order contributions is

$$
\begin{aligned}
\left(Z \Delta_{\omega \boldsymbol{k}}-\Delta_{\omega \boldsymbol{k}}^{\text {free }}\right)= & -\frac{\lambda^{2}}{24(2 \pi)^{2 d}} \int \frac{\mathrm{d}^{d} k_{1} \mathrm{~d}^{d} k_{2} \mathrm{~d}^{d} k_{3}}{\omega_{k_{1}} \omega_{k_{2}} \omega_{k_{3}}} \delta\left(\boldsymbol{k}_{1}+\boldsymbol{k}_{2}+\boldsymbol{k}_{3}\right) \\
& \times \frac{\omega_{k_{1}}+\omega_{k_{2}}+\omega_{k_{3}}}{\left[\left(\omega_{k_{1}}+\omega_{k_{2}}+\omega_{k_{3}}\right)^{2}-m^{2}\right]^{2}} \frac{1}{\left(\omega_{k_{1}}+\omega_{k_{2}}+\omega_{k_{3}}\right)^{2}-\left(\omega^{2}-k^{2}\right)} \\
& +\frac{2 \lambda^{3}}{64(2 \pi)^{3 d}} \int \frac{\mathrm{d}^{d} k_{1} \mathrm{~d}^{d} k_{2} \mathrm{~d}^{d} k_{3} \mathrm{~d}^{d} k_{4} \mathrm{~d}^{d} k_{1}^{\prime}}{\omega_{k_{1}} \omega_{k_{2}} \omega_{k_{3}} \omega_{k_{4}} \omega_{k_{1}^{\prime}}} \frac{\delta\left(\boldsymbol{k}_{1}+\boldsymbol{k}_{2}-\boldsymbol{k}_{3}-\boldsymbol{k}_{4}\right) \delta\left(\boldsymbol{k}_{1}+\boldsymbol{k}_{2}+\boldsymbol{k}_{1}^{\prime}\right)}{\left(\omega_{k_{1}}+\omega_{k_{2}}\right)^{2}-\left(\omega_{k_{3}}+\omega_{k_{4}}\right)^{2}} \\
& \times\left[\frac{\omega_{k_{3}}+\omega_{k_{4}}+\omega_{k_{1}^{\prime}}}{\left[\left(\omega_{k_{3}}+\omega_{k_{4}}+\omega_{k_{1}^{\prime}}\right)^{2}-m^{2}\right]^{2}} \frac{\omega_{k_{1}}+\omega_{k_{2}}}{\left(\omega_{k_{3}}+\omega_{k_{4}}+\omega_{k_{1}^{\prime}}\right)^{2}-\left(\omega^{2}-k^{2}\right)}\right. \\
& \left.-\frac{\omega_{k_{1}}+\omega_{k_{2}}+\omega_{k_{1}^{\prime}}}{\left[\left(\omega_{k_{1}}+\omega_{k_{2}}+\omega_{k_{1}^{\prime}}\right)^{2}-m^{2}\right]^{2}} \frac{\omega_{k_{3}}+\omega_{k_{4}}}{\left(\omega_{k_{1}}+\omega_{k_{2}}+\omega_{k_{1}^{\prime}}\right)^{2}-\left(\omega^{2}-k^{2}\right)}\right] .
\end{aligned}
$$


The corresponding renormalized expressions for $Z$ and $\lambda^{\prime}$ are shown in Eqs. (A4) and (A5), respectively. From these expressions, we observe that the previous $Z$ [Eq. (32)] is recovered for $m^{2}=0$, whereas the previous $\lambda^{\prime}$ [Eq. (33)] corresponds to the first-order expansion in $\mathrm{m}^{2}$, having no contribution from the connected-2 diagram (as we have already mentioned).

The beauty of the above regularization schemes is that they allow one to express $Z$ and $\lambda^{\prime}$ in a very elegant form by introducing two characteristic length scales: (a) a lowenergy length scale $\ell_{m}=1 / m$ as set by the physical mass $m$ and (b) a high-energy dissipative length scale $\ell_{\gamma}=\gamma^{1 / 3}$ as set by the friction parameter $\gamma$ [4] and by passing from sums to integrals with the rule proposed in Eq. (25). For example, using the renormalization scheme of Eq. (32) for the connection $Z$ between free particles and clouds, we find the following elegant equation:

$$
\begin{aligned}
Z= & 1+\frac{\lambda^{2}}{24(2 \pi)^{2 d}} I_{2}\left(\ell_{m}^{2 \varepsilon}-\ell_{\gamma}^{2 \varepsilon}\right) \\
& -\frac{\lambda^{3}}{64(2 \pi)^{3 d}} I_{3}\left(\ell_{m}^{3 \varepsilon}-\ell_{\gamma}^{3 \varepsilon}\right),
\end{aligned}
$$

where

$$
I_{2}=\int \frac{\mathrm{d}^{d} k_{1} \mathrm{~d}^{d} k_{2}}{k_{1} k_{2}\left|k_{1}+k_{2}\right|} \frac{1}{\left(k_{1}+k_{2}+\left|k_{1}+k_{2}\right|\right)^{3}},
$$

and

$$
\begin{aligned}
I_{3}= & \int \frac{\mathrm{d}^{d} k_{1} \mathrm{~d}^{d} k_{2} \mathrm{~d}^{d} k_{3}}{k_{1} k_{2} k_{3}\left|k_{1}+k_{3}\right|\left|k_{2}+k_{3}\right|} \\
& \times\left[\frac{1}{k_{1}+\left|k_{1}+k_{3}\right|-k_{2}-\left|k_{2}+k_{3}\right|}\left(\frac{1}{\left(k_{2}+\left|k_{2}+k_{3}\right|+k_{3}\right)^{3}}-\frac{1}{\left(k_{1}+\left|k_{1}+k_{3}\right|+k_{3}\right)^{3}}\right)\right. \\
& \left.+\frac{1}{k_{1}+\left|k_{1}+k_{3}\right|+k_{2}+\left|k_{2}+k_{3}\right|}\left(\frac{1}{\left(k_{2}+\left|k_{2}+k_{3}\right|+k_{3}\right)^{3}}+\frac{1}{\left(k_{1}+\left|k_{1}+k_{3}\right|+k_{3}\right)^{3}}\right)\right] .
\end{aligned}
$$

\section{CALCULATION OF $\lambda^{*}$}

In Sec. III, we derived the propagator of the dissipative quantum field theory up to third order in the interaction parameter $\lambda$, which in Sec. IV we proved to be compatible with the result from the Lagrangian approach in the limit of zero dissipation. Then, in Sec. V, we showed how one can regularize the result by properly defining the $Z$ and $\lambda^{\prime}$ parameters so that a finite expression is obtained. In this section, we see that the general expression for the third-order propagator leads naturally to the correct estimate of the critical value $\lambda^{*}$ of the theory known in the literature \{see for example Eq. (10.201) of [14]\}, which was not possible to check with the expression for the propagator up to second order [4].

The starting point is Eq. (24) without the connected-2 terms [the last line in Eq. (24)], because as we observed in Sec. V they drop out. We simplify the analysis by working in the limit $s \rightarrow 0$, corresponding to the long-time or lowenergy behavior. Then, Eq. (24) reduces to

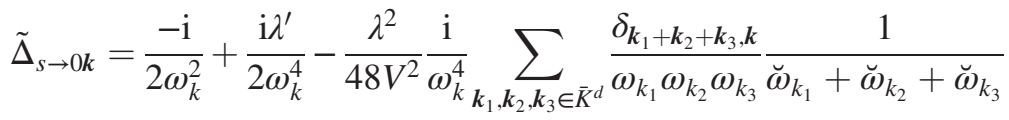

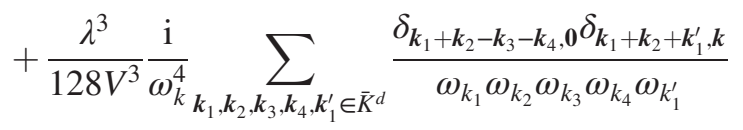

$$
\begin{aligned}
& \times\left[\frac{1}{\breve{\omega}_{k_{1}}+\breve{\omega}_{k_{2}}-\breve{\omega}_{k_{3}}-\breve{\omega}_{k_{4}}}\left(\frac{1}{\breve{\omega}_{k_{3}}+\breve{\omega}_{k_{4}}+\breve{\omega}_{k_{1}^{\prime}}}-\frac{1}{\breve{\omega}_{k_{1}}+\breve{\omega}_{k_{2}}+\breve{\omega}_{k_{1}^{\prime}}}\right)\right. \\
& \left.+\frac{1}{\breve{\omega}_{k_{1}}+\breve{\omega}_{k_{2}}+\breve{\omega}_{k_{3}}+\breve{\omega}_{k_{4}}}\left(\frac{1}{\breve{\omega}_{k_{3}}+\breve{\omega}_{k_{4}}+\breve{\omega}_{k_{1}^{\prime}}}+\frac{1}{\breve{\omega}_{k_{1}}+\breve{\omega}_{k_{2}}+\breve{\omega}_{k_{1}^{\prime}}}\right)\right] \text {. }
\end{aligned}
$$

The key observation to move on is that the product of the two loops in Fig. 2 over momentum $\boldsymbol{k}_{1}$ (or, equivalently, $\boldsymbol{k}_{2}$ ) and $\boldsymbol{k}_{3}$ (or, equivalently, $\boldsymbol{k}_{4}$ ) can be approximated as the square of a single loop. This observation is also reported in the literature for the two-loop contribution to the four-point or vertex function in the $D$-vector formalism \{see for example p. 40 in [2] and pp. 281-282 in [16]\}. We can clearly see this by redesigning the Feynman diagram shown in Fig. 2 in the form of the Feynman diagram shown in Fig. 5. 
Based on this observation, we replace the pair of dummy momenta $\left(\boldsymbol{k}_{\mathbf{3}}, \boldsymbol{k}_{\mathbf{4}}\right)$ with $\left(\boldsymbol{k}_{\mathbf{1}}, \boldsymbol{k}_{\mathbf{2}}\right)$ in the second, third, and fourth lines of Eq. (39). Then, we can notice that between the second- and third-order terms there is a common factor (after relabeling the momentum $\boldsymbol{k}_{3}$ with $\boldsymbol{k}_{1}^{\prime}$ in the secondorder term). Thus, Eq. (39) reduces to

$$
\begin{aligned}
\tilde{\Delta}_{s \rightarrow 0 k}= & \frac{-\mathrm{i}}{2 \omega_{k}^{2}}+\frac{\mathrm{i} \lambda^{\prime}}{2 \omega_{k}^{4}}-\frac{\lambda^{2}}{48 V^{2}} \frac{\mathrm{i}}{\omega_{k}^{4}} \sum_{\boldsymbol{k}_{1}, \boldsymbol{k}_{2}, \boldsymbol{k}_{1}^{\prime} \in \bar{K}^{d}} \frac{\delta_{\boldsymbol{k}_{1}+\boldsymbol{k}_{2}+\boldsymbol{k}_{1}^{\prime}, k}}{\omega_{k_{1}} \omega_{k_{2}} \omega_{k_{1}^{\prime}}} \frac{1}{\left[\left(\breve{\omega}_{k_{1}}+\breve{\omega}_{k_{2}}\right)+\breve{\omega}_{k_{1}^{\prime}}\right]} \\
& \times\left\{1-\lambda \frac{48}{128 V} \sum_{\boldsymbol{k}_{1}, k_{2} \in \bar{K}^{d}} \frac{\delta_{\boldsymbol{k}_{1}+\boldsymbol{k}_{2}+\boldsymbol{k}_{1}^{\prime}, k}}{\omega_{k_{1}} \omega_{k_{2}}} \frac{1}{\left(\breve{\omega}_{k_{1}}+\breve{\omega}_{k_{2}}\right)} \frac{2\left(\breve{\omega}_{k_{1}}+\breve{\omega}_{k_{2}}\right)+\breve{\omega}_{k_{1}^{\prime}}}{\left[\left(\breve{\omega}_{k_{1}}+\breve{\omega}_{k_{2}}\right)+\breve{\omega}_{k_{1}^{\prime}}\right]}\right\} .
\end{aligned}
$$

The nice thing about Eq. (40) is that it looks very much like the corresponding expression for the four-point correlation function (the vertex) elaborated in [4]. This is extremely beneficial because it allows us to extract the value of $\lambda^{*}$ by following a similar analysis as in [4]. For example, we can calculate the sums for any value of $\boldsymbol{k}_{1}$ or $\boldsymbol{k}_{2}$ (magnitudes from 0 to $\infty$ ) appearing in the last term inside the brackets in the last line of Eq. (40) by neglecting the contributions from the momenta $\boldsymbol{k}$ and $\boldsymbol{k}_{1}^{\prime}$ (fixed magnitudes) both in the $\delta$ function and in the expressions inside the sums,

$$
\begin{aligned}
& \lambda \frac{48}{128 V} \sum_{\boldsymbol{k}_{1}, k_{2} \in \bar{K}^{d}} \frac{\delta_{k_{1}+k_{2}+k_{1}^{\prime}, k}}{\omega_{k_{1}} \omega_{k_{2}}} \frac{1}{\left(\breve{\omega}_{k_{1}}+\breve{\omega}_{k_{2}}\right)} \frac{2\left(\breve{\omega}_{k_{1}}+\breve{\omega}_{k_{2}}\right)+\breve{\omega}_{k_{1}^{\prime}}}{\left[\left(\breve{\omega}_{k_{1}}+\breve{\omega}_{k_{2}}\right)+\breve{\omega}_{k_{1}^{\prime}}\right]} \\
& \left(\left\{k_{1}, k_{2}\right\} \gg\left\{k_{1}^{\prime}, k\right\}\right) \lambda \frac{48}{128 V} \sum_{\boldsymbol{k}_{1}, \boldsymbol{k}_{2} \in \bar{K}^{d}} \frac{\delta_{\boldsymbol{k}_{1}+\boldsymbol{k}_{2}, \mathbf{0}}}{\omega_{k_{1}} \omega_{k_{2}}} \frac{2\left(\breve{\omega}_{k_{1}}+\breve{\omega}_{k_{2}}\right)}{\left(\breve{\omega}_{k_{1}}+\breve{\omega}_{k_{2}}\right)^{2}} \\
& =\lambda \frac{48}{128 V} \sum_{\boldsymbol{k}_{1}, k_{2} \in \bar{K}^{d}} \frac{\delta_{\boldsymbol{k}_{1}+\boldsymbol{k}_{2}, \mathbf{0}}}{\omega_{k_{1}} \omega_{k_{2}}}\left[\frac{2\left(\omega_{k_{1}}+\omega_{k_{2}}\right)+2 \mathrm{i}\left(\gamma_{k_{1}}+\gamma_{k_{2}}\right)}{\left(\omega_{k_{1}}+\omega_{k_{2}}\right)^{2}+\left(\gamma_{k_{1}}+\gamma_{k_{2}}\right)^{2}}\right] \\
& =\lambda \frac{48}{128 V} \sum_{\boldsymbol{k}_{1}, \boldsymbol{k}_{2} \in \bar{K}^{d}} \frac{\delta_{\boldsymbol{k}_{1}+\boldsymbol{k}_{2}, \boldsymbol{0}}}{\omega_{k_{1}} \omega_{k_{2}}}\left(\frac{1}{\breve{\omega}_{k_{1}}+\breve{\omega}_{k_{2}}}+\frac{1}{\breve{\omega}_{k_{1}}^{*}+\breve{\omega}_{k_{2}}^{*}}\right) \text {, }
\end{aligned}
$$
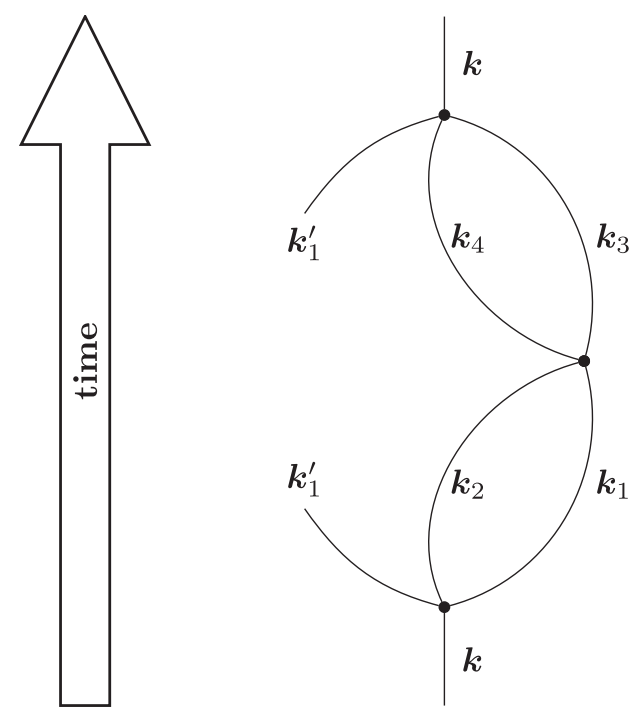

FIG. 5. Redesign of Fig. 2 describing the topology of the connected-1 Feynman diagram contributing to the propagator to third order in $\lambda$. where the SID assumption has been used when going from the third to the fourth lines (to get rid of the frictional part in the numerator). By applying the $\delta$ function in the last line of Eq. (41) and passing from sums to integrals using Eq. (25), we find

$$
\begin{aligned}
& \lambda \frac{48}{128(2 \pi)^{d}}\left[\int \mathrm{d}^{d} k_{1} \frac{1}{\omega_{k_{1}}^{3}}-\int \mathrm{d}^{d} k_{1} \frac{1}{\omega_{k_{1}}^{3}} \frac{\gamma_{k_{1}}^{2}}{\omega_{k_{1}}^{2}+\gamma_{k_{1}}^{2}}\right] \\
& =\lambda \frac{48}{128(2 \pi)^{d}}\left[\int \mathrm{d}^{d} k_{1} \frac{1}{\omega_{k_{1}}^{3}}-\ell_{\gamma}^{\varepsilon} \int \mathrm{d}^{d} q \frac{q^{3}}{1+q^{6}}\right],
\end{aligned}
$$

where we have also used the approximations $\omega_{k_{1}} \approx k_{1}$ and $\gamma_{k_{1}}=\gamma k_{1}^{4}$ for the large values of $k_{1}$ dominating the integral; we have also substituted $q=\ell_{\gamma} k_{1}$. Then, from Eq. (42) we identify \{see Eq. (1.95) and Eq. (2.76) in [4]\} $\lambda^{*}$ as

$$
\begin{aligned}
\frac{1}{\lambda^{*}} & =\frac{48}{128(2 \pi)^{d}}\left[\int \mathrm{d}^{d} q \frac{q^{3}}{1+q^{6}}\right] \\
\Rightarrow \lambda^{*} & =\frac{8}{3}(2 \pi)^{d}\left[\int \mathrm{d}^{d} q \frac{q^{3}}{1+q^{6}}\right]^{-1},
\end{aligned}
$$

which perfectly agrees with [4] [see, e.g., Eq. (2.77)], and thus, for small $\varepsilon$, we obtain [4]

$$
\lambda^{*} \approx \frac{16}{3} \pi^{2} \varepsilon
$$

\section{SUMMARY AND DISCUSSION}

Within the context of the dissipative quantum field theory developed in [4], we presented a perturbation expansion of the two-point correlation function (the propagator) of the scalar $\varphi^{4}$ theory up to third order in the interaction parameter $\lambda$, in the limit of zero temperature. The calculations were carried out with two different perturbation schemes, both of which gave identical results. In both methods, we relied on symbolic calculations for the handling of the operations needed in the intermediate calculations. The expression for the third-order contribution, in particular, contains four blocks of terms, each one comprising three types of diagrams: (i) connected-1, (ii) connected-2, and (iii) unconnected. The terms that 
correspond to the unconnected Feynman diagram do not contribute.

In the limit of vanishing dissipation (where complex frequencies are replaced by real frequencies), our expression leads self-consistently to the result of the Lagrangian quantum field theory upon integrating out the time or energy component.

The resulting expression for the propagator was further regularized by choosing properly the parameters $Z$ and $\lambda^{\prime}$ of the dissipative theory, and it was confirmed that in the continuum limit (limit of infinite volume where sums over momentum vectors are replaced by integrals) no divergences appear for vanishing friction.

Moreover, the theory produces the correct result for the critical coupling constant or interaction parameter $\lambda^{*}$ as a function of space dimensionality $d$.

\section{ACKNOWLEDGMENTS}

The authors are grateful to Alberto Montefusco for developing the very first version of the code used in the symbolic calculations of the present work. Very useful discussions with Prof. Mikhail Kompaniets on specific points of this work are deeply appreciated and gratefully acknowledged. P. V. Alatas acknowledges financial support from the Hellenic Foundation for Research and Innovation (HFRI/ELIDEK) through the 1st ELIDEK scholarship call for doctoral candidates (code: 2694).

\section{APPENDIX: REGULARIZATION}

In this Appendix, we present more details on the regularization procedure discussed in Sec. V. More specifically, we report the intermediate expressions between Eqs. (33) and (34), as well as the expressions which are accompanied by Eq. (35).

Starting from Eq. (30), using the expressions for $\tilde{\Delta}_{s k}\left[\right.$ Eq. (24)] and $\tilde{\Delta}_{s k}^{\text {free }}\left(=(-\mathrm{i}) /\left[2\left(s^{2}+\omega_{k}^{2}\right)\right]\right)$, inserting Eqs. (32) and (33) (the resulting expressions for $Z$ and $\lambda^{\prime}$, respectively) and considering the expansion in Eq. (31), we obtain for $\gamma=0$,

$$
\begin{aligned}
\left(s^{2}+\omega_{k}^{2}\right)^{2}\left(Z \tilde{\Delta}_{s k}-\tilde{\Delta}_{s \boldsymbol{k}}^{\mathrm{free}}\right)= & \frac{\lambda^{2}}{48 V^{2}} \sum_{\boldsymbol{k}_{1}, \boldsymbol{k}_{2}, \boldsymbol{k}_{3} \in \bar{K}^{d}} \frac{\mathrm{i}}{\omega_{k_{1}} \omega_{k_{2}} \omega_{k_{3}}}\left[\frac{\delta_{\boldsymbol{k}_{1}+\boldsymbol{k}_{2}+\boldsymbol{k}_{3}, \mathbf{0}}-\delta_{\boldsymbol{k}_{1}+\boldsymbol{k}_{2}+\boldsymbol{k}_{3}, \boldsymbol{k}}}{\omega_{k_{1}}+\omega_{k_{2}}+\omega_{k_{3}}}-\frac{\left(k^{2}+s^{2}\right) \delta_{\boldsymbol{k}_{1}+\boldsymbol{k}_{2}+\boldsymbol{k}_{3}, \mathbf{0}}-s^{2} \delta_{\boldsymbol{k}_{1}+\boldsymbol{k}_{2}+\boldsymbol{k}_{3}, \boldsymbol{k}}}{\left(\omega_{k_{1}}+\omega_{k_{2}}+\omega_{k_{3}}\right)^{3}}\right] \\
& -\frac{\lambda^{3}}{128 V^{3}} \sum_{\boldsymbol{k}_{1}, \boldsymbol{k}_{2}, \boldsymbol{k}_{3}, \boldsymbol{k}_{4}, \boldsymbol{k}_{1}^{\prime} \in \bar{K}^{d}} \frac{\mathrm{i}}{\omega_{k_{1}} \omega_{k_{2}} \omega_{k_{3}} \omega_{k_{4}} \omega_{k_{1}^{\prime}}} \delta_{\boldsymbol{k}_{1}+\boldsymbol{k}_{2}-\boldsymbol{k}_{3}-\boldsymbol{k}_{4}, \mathbf{0}} \\
& \times\left[\frac{\delta_{\boldsymbol{k}_{1}+\boldsymbol{k}_{2}+\boldsymbol{k}_{1}^{\prime}, \mathbf{0}}-\delta_{\boldsymbol{k}_{1}+\boldsymbol{k}_{2}+\boldsymbol{k}_{1}^{\prime}, \boldsymbol{k}}}{\omega_{k_{1}}+\omega_{k_{2}}-\omega_{k_{3}}-\omega_{k_{4}}}\left(\frac{1}{\omega_{k_{3}}+\omega_{k_{4}}+\omega_{k_{1}^{\prime}}}-\frac{1}{\omega_{k_{1}}+\omega_{k_{2}}+\omega_{k_{1}^{\prime}}}\right)\right. \\
& +\frac{\delta_{\boldsymbol{k}_{1}+\boldsymbol{k}_{2}+\boldsymbol{k}_{1}^{\prime}, \mathbf{0}}-\delta_{\boldsymbol{k}_{1}+\boldsymbol{k}_{2}+\boldsymbol{k}_{1}^{\prime}, \boldsymbol{k}}}{\omega_{k_{1}}+\omega_{k_{2}}+\omega_{k_{3}}+\omega_{k_{4}}}\left(\frac{1}{\omega_{k_{3}}+\omega_{k_{4}}+\omega_{k_{1}^{\prime}}}+\frac{1}{\omega_{k_{1}}+\omega_{k_{2}}+\omega_{k_{1}^{\prime}}}\right) \\
& -\frac{\left(k^{2}+s^{2}\right) \delta_{\boldsymbol{k}_{1}+\boldsymbol{k}_{2}+\boldsymbol{k}_{1}^{\prime}, \mathbf{0}}-s^{2} \delta_{\boldsymbol{k}_{1}+\boldsymbol{k}_{2}+\boldsymbol{k}_{1}^{\prime}, \boldsymbol{k}}}{\omega_{k_{1}}+\omega_{k_{2}}-\omega_{k_{3}}-\omega_{k_{4}}}\left(\frac{1}{\left(\omega_{k_{3}}+\omega_{k_{4}}+\omega_{k_{1}^{\prime}}\right)^{3}}-\frac{1}{\left(\omega_{k_{1}}+\omega_{k_{2}}+\omega_{k_{1}^{\prime}}\right)^{3}}\right) \\
& \left.-\frac{\left(k^{2}+s^{2}\right) \delta_{\boldsymbol{k}_{1}+\boldsymbol{k}_{2}+\boldsymbol{k}_{1}^{\prime}, \mathbf{0}}-s^{2} \delta_{\boldsymbol{k}_{1}+\boldsymbol{k}_{2}+\boldsymbol{k}_{1}^{\prime}, \boldsymbol{k}}}{\omega_{k_{1}}+\omega_{k_{2}}+\omega_{k_{3}}+\omega_{k_{4}}}\left(\frac{1}{\left(\omega_{k_{3}}+\omega_{k_{4}}+\omega_{k_{1}^{\prime}}\right)^{3}}+\frac{1}{\left(\omega_{k_{1}}+\omega_{k_{2}}+\omega_{k_{1}^{\prime}}\right)^{3}}\right)\right] .
\end{aligned}
$$

The terms proportional to $\left(\omega_{k_{a}}+\omega_{k_{b}}+\omega_{k_{c}}\right)^{-1}$ lead to a power-law divergence for $d>2$, while the terms proportional to $\left(\omega_{k_{a}}+\omega_{k_{b}}+\omega_{k_{c}}\right)^{-3}$ lead to a logarithmic divergence for $d=3$. Thus, to avoid divergences, the sums

$$
\sum_{\boldsymbol{k}_{1}, \boldsymbol{k}_{2}, \boldsymbol{k}_{3} \in \bar{K}^{3}} \frac{\mathrm{i}}{\omega_{k_{1}} \omega_{k_{2}} \omega_{k_{3}}}\left[\frac{\delta_{\boldsymbol{k}_{1}+\boldsymbol{k}_{2}+\boldsymbol{k}_{3}, \mathbf{0}}-\delta_{\boldsymbol{k}_{1}+\boldsymbol{k}_{2}+\boldsymbol{k}_{3}, \boldsymbol{k}}}{\omega_{k_{1}}+\omega_{k_{2}}+\omega_{k_{3}}}-\frac{k^{2} \delta_{\boldsymbol{k}_{1}+\boldsymbol{k}_{2}+\boldsymbol{k}_{3}, \mathbf{0}}}{\left(\omega_{k_{1}}+\omega_{k_{2}}+\omega_{k_{3}}\right)^{3}}\right],
$$

and 


$$
\begin{aligned}
& \sum_{\boldsymbol{k}_{1}, \boldsymbol{k}_{2}, \boldsymbol{k}_{3}, \boldsymbol{k}_{4}, \boldsymbol{k}_{1}^{\prime} \in \bar{K}^{3}} \frac{\mathrm{i}}{\omega_{k_{1}} \omega_{k_{2}} \omega_{k_{3}} \omega_{k_{4}} \omega_{k_{1}^{\prime}}} \delta_{\boldsymbol{k}_{1}+\boldsymbol{k}_{2}-\boldsymbol{k}_{3}-\boldsymbol{k}_{4}, \mathbf{0}} \\
& \times\left[\frac{\delta_{\boldsymbol{k}_{1}+\boldsymbol{k}_{2}+\boldsymbol{k}_{1}^{\prime}, \mathbf{0}}-\delta_{\boldsymbol{k}_{1}+\boldsymbol{k}_{2}+\boldsymbol{k}_{1}^{\prime}, \boldsymbol{k}}}{\omega_{k_{1}}+\omega_{k_{2}}-\omega_{k_{3}}-\omega_{k_{4}}}\left(\frac{1}{\omega_{k_{3}}+\omega_{k_{4}}+\omega_{k_{1}^{\prime}}}-\frac{1}{\omega_{k_{1}}+\omega_{k_{2}}+\omega_{k_{1}^{\prime}}}\right)\right. \\
& -\frac{k^{2} \delta_{\boldsymbol{k}_{1}+\boldsymbol{k}_{2}+\boldsymbol{k}_{1}^{\prime}, \mathbf{0}}}{\omega_{k_{1}}+\omega_{k_{2}}-\omega_{k_{3}}-\omega_{k_{4}}}\left(\frac{1}{\left(\omega_{k_{3}}+\omega_{k_{4}}+\omega_{k_{1}^{\prime}}\right)^{3}}-\frac{1}{\left(\omega_{k_{1}}+\omega_{k_{2}}+\omega_{k_{1}^{\prime}}\right)^{3}}\right) \\
& +\frac{\delta_{\boldsymbol{k}_{1}+\boldsymbol{k}_{2}+\boldsymbol{k}_{1}^{\prime}, \mathbf{0}}-\delta_{\boldsymbol{k}_{1}+\boldsymbol{k}_{2}+\boldsymbol{k}_{1}^{\prime}, \boldsymbol{k}}}{\omega_{k_{1}}+\omega_{k_{2}}+\omega_{k_{3}}+\omega_{k_{4}}}\left(\frac{1}{\omega_{k_{3}}+\omega_{k_{4}}+\omega_{k_{1}^{\prime}}}+\frac{1}{\omega_{k_{1}}+\omega_{k_{2}}+\omega_{k_{1}^{\prime}}}\right) \\
& \left.-\frac{k^{2} \delta_{\boldsymbol{k}_{1}+\boldsymbol{k}_{2}+\boldsymbol{k}_{1}^{\prime}, \mathbf{0}}}{\omega_{k_{1}}+\omega_{k_{2}}+\omega_{k_{3}}+\omega_{k_{4}}}\left(\frac{1}{\left(\omega_{k_{3}}+\omega_{k_{4}}+\omega_{k_{1}^{\prime}}\right)^{3}}+\frac{1}{\left(\omega_{k_{1}}+\omega_{k_{2}}+\omega_{k_{1}^{\prime}}\right)^{3}}\right)\right],
\end{aligned}
$$

must remain finite for an infinitely large lattice $\bar{K}^{3}$ of momentum vectors in $d=3$ dimensions. It can be shown that this is, indeed, the case through a numerical calculation.

In fact, one can perform a more elegant analysis by passing from sums to integrals using the rule shown in Eq. (25), and from the Kronecker $\delta$ symbol to the Dirac $\delta$ function with the help of Eq. (26b), to find (for $\boldsymbol{k}=\mathbf{0}$ )

$$
\begin{aligned}
& \left(Z \Delta_{\omega \boldsymbol{k}}-\Delta_{\omega \boldsymbol{k}}^{\mathrm{free}}\right)=\frac{\lambda^{2}}{24(2 \pi)^{2 d}} \frac{1}{\left(\omega^{2}-\omega_{k}^{2}\right)^{2}} \int \frac{\mathrm{d}^{d} k_{1} \mathrm{~d}^{d} k_{2} \mathrm{~d}^{d} k_{3}}{\omega_{k_{1}} \omega_{k_{2}} \omega_{k_{3}}} \delta\left(\boldsymbol{k}_{1}+\boldsymbol{k}_{2}+\boldsymbol{k}_{3}\right) \\
& \times\left[\frac{1}{\omega_{k_{1}}+\omega_{k_{2}}+\omega_{k_{3}}}+\frac{\omega^{2}-k^{2}}{\left(\omega_{k_{1}}+\omega_{k_{2}}+\omega_{k_{3}}\right)^{3}}-\frac{\omega_{k_{1}}+\omega_{k_{2}}+\omega_{k_{3}}}{\left(\omega_{k_{1}}+\omega_{k_{2}}+\omega_{k_{3}}\right)^{2}-\left(\omega^{2}-k^{2}\right)}\right] \\
& -\frac{1}{\left(\omega^{2}-\omega_{k}^{2}\right)^{2}} \frac{\lambda^{3}}{64(2 \pi)^{3 d}} \int \frac{\mathrm{d}^{d} k_{1} \mathrm{~d}^{d} k_{2} \mathrm{~d}^{d} k_{3} \mathrm{~d}^{d} k_{4} \mathrm{~d}^{d} k_{1}^{\prime}}{\omega_{k_{1}} \omega_{k_{2}} \omega_{k_{3}} \omega_{k_{4}} \omega_{k_{1}^{\prime}}} \delta\left(\boldsymbol{k}_{1}+\boldsymbol{k}_{2}-\boldsymbol{k}_{3}-\boldsymbol{k}_{4}\right) \delta\left(\boldsymbol{k}_{1}+\boldsymbol{k}_{2}+\boldsymbol{k}_{1}^{\prime}\right) \\
& \times\left[\frac{1}{\omega_{k_{1}}+\omega_{k_{2}}-\omega_{k_{3}}-\omega_{k_{4}}}\left(\frac{1}{\omega_{k_{3}}+\omega_{k_{4}}+\omega_{k_{1}^{\prime}}}-\frac{1}{\omega_{k_{1}}+\omega_{k_{2}}+\omega_{k_{1}^{\prime}}}\right)\right. \\
& +\frac{1}{\omega_{k_{1}}+\omega_{k_{2}}+\omega_{k_{3}}+\omega_{k_{4}}}\left(\frac{1}{\omega_{k_{3}}+\omega_{k_{4}}+\omega_{k_{1}^{\prime}}}+\frac{1}{\omega_{k_{1}}+\omega_{k_{2}}+\omega_{k_{1}^{\prime}}}\right) \\
& +\frac{\left(\omega^{2}-k^{2}\right)}{\omega_{k_{1}}+\omega_{k_{2}}-\omega_{k_{3}}-\omega_{k_{4}}}\left(\frac{1}{\left(\omega_{k_{3}}+\omega_{k_{4}}+\omega_{k_{1}^{\prime}}\right)^{3}}-\frac{1}{\left(\omega_{k_{1}}+\omega_{k_{2}}+\omega_{k_{1}^{\prime}}\right)^{3}}\right) \\
& +\frac{\left(\omega^{2}-k^{2}\right)}{\omega_{k_{1}}+\omega_{k_{2}}+\omega_{k_{3}}+\omega_{k_{4}}}\left(\frac{1}{\left(\omega_{k_{3}}+\omega_{k_{4}}+\omega_{k_{1}^{\prime}}\right)^{3}}+\frac{1}{\left(\omega_{k_{1}}+\omega_{k_{2}}+\omega_{k_{1}^{\prime}}\right)^{3}}\right) \\
& -\frac{1}{\omega_{k_{1}}+\omega_{k_{2}}-\omega_{k_{3}}-\omega_{k_{4}}}\left(\frac{\omega_{k_{3}}+\omega_{k_{4}}+\omega_{k_{1}^{\prime}}}{\left(\omega_{k_{3}}+\omega_{k_{4}}+\omega_{k_{1}^{\prime}}\right)^{2}-\left(\omega^{2}-k^{2}\right)}-\frac{\omega_{k_{1}}+\omega_{k_{2}}+\omega_{k_{1}^{\prime}}}{\left(\omega_{k_{1}}+\omega_{k_{2}}+\omega_{k_{1}^{\prime}}\right)^{2}-\left(\omega^{2}-k^{2}\right)}\right) \\
& \left.-\frac{1}{\omega_{k_{1}}+\omega_{k_{2}}+\omega_{k_{3}}+\omega_{k_{4}}}\left(\frac{\omega_{k_{3}}+\omega_{k_{4}}+\omega_{k_{1}^{\prime}}}{\left(\omega_{k_{3}}+\omega_{k_{4}}+\omega_{k_{1}^{\prime}}\right)^{2}-\left(\omega^{2}-k^{2}\right)}+\frac{\omega_{k_{1}}+\omega_{k_{2}}+\omega_{k_{1}^{\prime}}}{\left(\omega_{k_{1}}+\omega_{k_{2}}+\omega_{k_{1}^{\prime}}\right)^{2}-\left(\omega^{2}-k^{2}\right)}\right)\right] \text {, }
\end{aligned}
$$

which can be further simplified to Eq. (34).

In view of the "on shell renormalization", one can expand not with respect to $\omega^{2}-k^{2}$ but with respect to $\omega^{2}-k^{2}-m^{2}$ when going from Eq. (A3) to Eq. (34). The resulting new expressions for $Z$ and $\lambda^{\prime}$ are 


$$
\begin{aligned}
Z= & 1+\frac{\lambda^{2}}{24 V^{2}} \sum_{k_{1}, k_{2}, k_{3} \in K^{d}} \frac{\delta_{k_{1}+k_{2}+k_{3}, 0}}{\omega_{k_{1}} \omega_{k_{2}} \omega_{k_{3}}} \frac{\breve{\omega}_{k_{1}}+\breve{\omega}_{k_{2}}+\breve{\omega}_{k_{3}}}{\left[\left(\breve{\omega}_{k_{1}}+\breve{\omega}_{k_{2}}+\breve{\omega}_{k_{3}}\right)^{2}-m^{2}\right]^{2}} \\
& -\frac{\lambda^{3}}{64 V^{3}} \sum_{k_{1}, k_{2}, k_{3}, k_{4}, k_{1}^{\prime} \in K^{d}} \frac{\delta_{k_{1}+k_{2}-k_{3}-k_{4}, 0} \delta_{k_{1}+k_{2}+k_{1}^{\prime}, 0}}{\omega_{k_{1}} \omega_{k_{2}} \omega_{k_{3}} \omega_{k_{4}} \omega_{k_{1}^{\prime}}} \\
& \times\left[\frac{1}{\breve{\omega}_{k_{1}}+\breve{\omega}_{k_{2}}-\breve{\omega}_{k_{3}}-\breve{\omega}_{k_{4}}}\left(\frac{\breve{\omega}_{k_{3}}+\breve{\omega}_{k_{4}}+\breve{\omega}_{k_{1}^{\prime}}}{\left[\left(\breve{\omega}_{k_{3}}+\breve{\omega}_{k_{4}}+\breve{\omega}_{k_{1}^{\prime}}\right)^{2}-m^{2}\right]^{2}}-\frac{\breve{\omega}_{k_{1}}+\breve{\omega}_{k_{2}}+\breve{\omega}_{k_{1}^{\prime}}}{\left[\left(\breve{\omega}_{k_{1}}+\breve{\omega}_{k_{2}}+\breve{\omega}_{k_{1}^{\prime}}\right)^{2}-m^{2}\right]^{2}}\right)\right. \\
& +\frac{1}{\breve{\omega}_{k_{1}}+\breve{\omega}_{k_{2}}+\breve{\omega}_{k_{3}}+\breve{\omega}_{k_{4}}}\left(\frac{\breve{\omega}_{k_{3}}+\breve{\omega}_{k_{4}}+\breve{\omega}_{k_{1}^{\prime}}}{\left[\left(\breve{\omega}_{k_{3}}+\breve{\omega}_{k_{4}}+\breve{\omega}_{k_{1}^{\prime}}\right)^{2}-m^{2}\right]^{2}}+\frac{\breve{\omega}_{k_{2}}+\breve{\omega}_{k_{1}^{\prime}}}{\left.\left.\left[\left(\breve{\omega}_{k_{1}}+\breve{\omega}_{k_{2}}+\breve{\omega}_{k_{1}^{\prime}}\right)^{2}-m^{2}\right]^{2}\right)\right],}\right.
\end{aligned}
$$

and

$$
\begin{aligned}
& \lambda^{\prime}=\frac{\lambda^{2}}{24 V^{2}} \sum_{k_{1}, k_{2}, k_{3} \in K^{d}} \frac{\delta_{k_{1}+k_{2}+k_{3}, 0}}{\omega_{k_{1}} \omega_{k_{2}} \omega_{k_{3}}} \frac{\breve{\omega}_{k_{1}}+\breve{\omega}_{k_{2}}+\breve{\omega}_{k_{3}}}{\left(\breve{\omega}_{k_{1}}+\breve{\omega}_{k_{2}}+\breve{\omega}_{k_{3}}\right)^{2}-m^{2}} \\
& -\frac{\lambda^{3}}{64 V^{3}} \sum_{\boldsymbol{k}_{1}, \boldsymbol{k}_{2}, \boldsymbol{k}_{3}, \boldsymbol{k}_{4}, \boldsymbol{k}_{1}^{\prime} \in K^{d}} \frac{\delta_{\boldsymbol{k}_{1}+\boldsymbol{k}_{2}-\boldsymbol{k}_{3}-\boldsymbol{k}_{4}, \mathbf{0}} \delta_{\boldsymbol{k}_{1}+\boldsymbol{k}_{2}+\boldsymbol{k}_{1}^{\prime}, \mathbf{0}}}{\omega_{k_{1}} \omega_{k_{2}} \omega_{k_{3}} \omega_{k_{4}} \omega_{k_{1}^{\prime}}} \\
& \times\left[\frac{1}{\breve{\omega}_{k_{1}}+\breve{\omega}_{k_{2}}-\breve{\omega}_{k_{3}}-\breve{\omega}_{k_{4}}}\left(\frac{\breve{\omega}_{k_{3}}+\breve{\omega}_{k_{4}}+\breve{\omega}_{k_{1}^{\prime}}}{\left(\breve{\omega}_{k_{3}}+\breve{\omega}_{k_{4}}+\breve{\omega}_{k_{1}^{\prime}}\right)^{2}-m^{2}}-\frac{\breve{\omega}_{k_{1}}+\breve{\omega}_{k_{2}}+\breve{\omega}_{k_{1}^{\prime}}}{\left(\breve{\omega}_{k_{1}}+\breve{\omega}_{k_{2}}+\breve{\omega}_{k_{1}^{\prime}}\right)^{2}-m^{2}}\right)\right. \\
& \left.+\frac{1}{\breve{\omega}_{k_{1}}+\breve{\omega}_{k_{2}}+\breve{\omega}_{k_{3}}+\breve{\omega}_{k_{4}}}\left(\frac{\breve{\omega}_{k_{3}}+\breve{\omega}_{k_{4}}+\breve{\omega}_{k_{1}^{\prime}}}{\left(\breve{\omega}_{k_{3}}+\breve{\omega}_{k_{4}}+\breve{\omega}_{k_{1}^{\prime}}\right)^{2}-m^{2}}+\frac{\breve{\omega}_{k_{1}}+\breve{\omega}_{k_{2}}+\breve{\omega}_{k_{1}^{\prime}}}{\left(\breve{\omega}_{k_{1}}+\breve{\omega}_{k_{2}}+\breve{\omega}_{k_{1}^{\prime}}\right)^{2}-m^{2}}\right)\right] \text {. }
\end{aligned}
$$

The final expression for the second- and third-order contributions to the propagator under the "on shell renormalization" scheme is shown in Eq. (35) of the main text.

[1] K. G. Wilson and J. Kogut, Phys. Rep. 12, 75 (1974).

[2] M. E. Peskin and D. V. Schroeder, An Introduction to Quantum Field Theory (Perseus Books, Reading, MA, 1995).

[3] S. Weinberg, The Quantum Theory of Fields, Volume 2: Modern Applications (Cambridge University Press, Cambridge, England, 2005).

[4] H. C. Öttinger, A Philosophical Approach to Quantum Field Theory (Cambridge University Press, Cambridge, England, 2017).

[5] H. C. Öttinger, Phys. Rev. E 79, 021124 (2009).

[6] A. Oldofredi and H. C. Öttinger, Eur. J. Phil. Sci. 11, 18 (2021).

[7] H. P. Breuer and F. Petruccione, The Theory of Open Quantum Systems (Oxford University Press, Great Clarendon Street, 2002).

[8] U. Weiss, Quantum Dissipative Systems 4th Edition. (World Scientific Publishing Company, Singapore, 2012).
[9] H. Grabert, Projection Operator Techniques in Nonequilibrium Statistical Mechanics (Springer Berlin Heidelberg, Berlin, Heidelberg, 1982).

[10] H. C. Öttinger, Phys. Rev. D 84, 065007 (2011).

[11] D. Taj and H. C. Öttinger, Phys. Rev. A 92, 062128 (2015).

[12] G. Lindblad, Commun. Math. Phys. 48, 119 (1976).

[13] C. Burgess and G. Moore, The Standard Model: A Primer (Cambridge University Press, Cambridge, England, 2006).

[14] H. Kleinert and V. Schulte-Frohlinde, Critical Properties of $\phi^{4}$-Theories (World Scientific, Singapore, 2001).

[15] P. R. S. Carvalho and M. M. Leite, J. Math. Phys. (N.Y.) 54, 093301 (2013).

[16] J. Zinn-Justin, Quantum Field Theory and Critical Phenomena, 4th ed., International Series of Monographs on Physics Vol. 13 (Oxford University Press, New York, 2002). 